TRANSACTIONS OF THE

AMERICAN MATHEMATICAL SOCIETY

Volume 361, Number 9, September 2009, Pages 4721-4749

S 0002-9947(09)04840-5

Article electronically published on March 19, 2009

\title{
SELF DELTA-EQUIVALENCE FOR LINKS WHOSE MILNOR'S ISOTOPY INVARIANTS VANISH
}

\author{
AKIRA YASUHARA
}

Dedicated to Professor Tetsuo Shibuya on his 60th birthday.

\begin{abstract}
For an $n$-component link, Milnor's isotopy invariants are defined for each multi-index $I=i_{1} i_{2} \ldots i_{m}\left(i_{j} \in\{1, \ldots, n\}\right)$. Here $m$ is called the length. Let $r(I)$ denote the maximum number of times that any index appears in $I$. It is known that Milnor invariants with $r=1$, i.e., Milnor invariants for all multi-indices $I$ with $r(I)=1$, are link-homotopy invariant. N. Habegger and X. S. Lin showed that two string links are link-homotopic if and only if their Milnor invariants with $r=1$ coincide. This gives us that a link in $S^{3}$ is linkhomotopic to a trivial link if and only if all Milnor invariants of the link with $r=1$ vanish. Although Milnor invariants with $r=2$ are not link-homotopy invariants, T. Fleming and the author showed that Milnor invariants with $r \leq 2$ are self $\Delta$-equivalence invariants. In this paper, we give a self $\Delta$-equivalence classification of the set of $n$-component links in $S^{3}$ whose Milnor invariants with length $\leq 2 n-1$ and $r \leq 2$ vanish. As a corollary, we have that a link is self $\Delta$-equivalent to a trivial link if and only if all Milnor invariants of the link with $r \leq 2$ vanish. This is a geometric characterization for links whose Milnor invariants with $r \leq 2$ vanish. The chief ingredient in our proof is Habiro's clasper theory. We also give an alternate proof of a link-homotopy classification of string links by using clasper theory.
\end{abstract}

\section{INTRODUCTION}

For an $n$-component link $L$, the Milnor invariant $\bar{\mu}_{L}(I)$ is defined for each multiindex $I=i_{1} i_{2} \ldots i_{m}\left(i_{j} \in\{1, \ldots, n\}\right)\left[18\right.$, 19]. Here $m$ is called the length of $\bar{\mu}_{L}(I)$ and denoted by $|I|$. Let $r(I)$ denote the maximum number of times that any index appears in $I$. For example, $r(1123)=2, r(1231223)=3$. It is known that if $r(I)=1$, then $\bar{\mu}_{L}(I)$ is a link-homotopy invariant [18], where link-homotopy is an equivalence relation on links generated by self-crossing changes. Similarly, for a string link $L$, the Milnor invariant $\mu_{L}(I)$ is defined [9]. While Milnor invariants are not strong enough to give a link-homotopy classification for links, they are complete for string links. In fact, the following is given by N. Habegger and X. S. Lin 9].

Received by the editors July 17, 2007.

2000 Mathematics Subject Classification. Primary 57M25, 57M27.

Key words and phrases. $\Delta$-move, self $\Delta$-move, $C_{n}$-move, link-homotopy, self $\Delta$-equivalence, Milnor invariant, string link, Brunnian link, clasper.

The author was partially supported by a Grant-in-Aid for Scientific Research (C) (\#18540071) of the Japan Society for the Promotion of Science.

(C)2009 American Mathematical Society Reverts to public domain 28 years from publication 


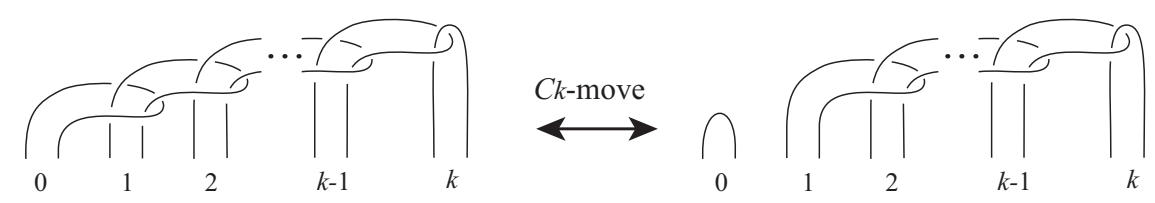

Figure 1.1. A $C_{k}$-move involves $k+1$ strands of a link

Theorem $1.1([9])$. Two $n$-component string links $L$ and $L^{\prime}$ are link-homotopic if and only if $\mu_{L}(I)=\mu_{L^{\prime}}(I)$ for any $I$ with $r(I)=1$.

We will give an alternate proof in section 4 via clasper theory. Actually we will give representatives determined by Milnor link-homotopy invariants for the linkhomotopy classes explicitly; see Theorem 4.3. As a corollary, we have that for $n$-component string links $L$ and $L^{\prime}$, and for a positive integer $k(k \leq n), \mu_{L}(I)=$ $\mu_{L^{\prime}}(I)$ for any $I$ with $r(I)=1$ and $|I| \leq k$ if and only if $L$ and $L^{\prime}$ are transformed into each other by combining link-homotopies and $C_{k}$-moves; see Corollary $4.5 \mathrm{~A}$ $C_{k}$-move is a local move on (string) links as illustrated in Figure 1.1. (A $C_{1}$-move is defined as the crossing change.) These local moves were introduced by Habiro [10. The $C_{k}$-move generates an equivalence relation on (string) links, called the $C_{k}$-equivalence.

For a string link $L$, let $\operatorname{cl}(L)$ denote the closure of $L$. It follows from the definitions that $\mu_{L}(I)=\bar{\mu}_{\mathrm{cl}(L)}(I)$ if $\mu_{L}(J)=0$ for any $J$ with $|J|<|I|$. Since the Milnor invariants of trivial (string) links are 0 , this and Theorem 1.1 imply the following. The theorem below is also shown by J. Milnor [18].

Theorem $1.2([18,9])$. A link $L$ in $S^{3}$ is link-homotopic to a trivial link if and only if $\bar{\mu}_{L}(I)=0$ for any $I$ with $r(I)=1$.

Although Milnor invariants with $r \geq 2$, i.e., Milnor invariants for all multi-index $I$ with $r(I) \geq 2$, are not necessarily link-homotopy invariants, they are generalized link-homotopy invariants. In fact, Fleming and the author 4 showed that Milnor invariants with $r \leq k$ are self $C_{k}$-equivalence invariants, where the self $C_{k^{-}}$ equivalence is an equivalence relation on (string) links generated by self $C_{k}$-moves, and a self $C_{k}$-move is a $C_{k}$-move where all the strands belong to the same component of a (string) link [26].

The $C_{k}$-move can also be defined by using the theory of claspers (see section 2). The (self) $C_{n}$-equivalence relation becomes finer as $n$ increases, i.e., the (self) $C_{m^{-}}$ equivalence implies the (self) $C_{k}$-equivalence for $m>k$. We remark that a (self) $C_{2}$-move is equivalent to a (self) $\Delta$-move defined by [21]. The $\Delta$-move is defined as a local move as illustrated in Figure 1.2 . We call the (self) $C_{2}$-equivalence the (self) $\Delta$-equivalence.

A self $\Delta$-equivalence classification of 2-component links was shown by Y. Nakanishi and Y. Ohyama [22]. It is still open for links with at least 3 components. Here we give the following theorem.

Theorem 1.3. Let $L$ and $L^{\prime}$ be n-component links. Suppose that $\bar{\mu}_{L}(I)=\bar{\mu}_{L^{\prime}}(I)=$ 0 for any $I$ with $|I| \leq 2 n-1$ and $r(I) \leq 2$. Then $L$ and $L^{\prime}$ are self $\Delta$-equivalent if and only if $\bar{\mu}_{L}(J)=\bar{\mu}_{L^{\prime}}(J)$ for any $J$ with $|J|=2 n$ and $r(J)=2$. 
Remark 1.4. (1) The 'only if' part follows directly from the fact that Milnor invariants with $r \leq k$ are self $C_{k}$-equivalence invariants 4 .

(2) In the last section, we characterize $n$-component links whose Milnor invariants of length $\leq 2 n-1$ and $r \leq 2$ vanish. More precisely, the Milnor invariants of an $n$-component link with length $\leq 2 n-1$ and $r \leq 2$ vanish if and only if, for any integer $i$ in $\{1, \ldots, n\}$, it is self $\Delta$-equivalent to a Brunnian link $L_{i}$ such that the $i$ th component $K$ of $L_{i}$ is null-homotopic in $S^{3} \backslash\left(L_{i}-K\right)$ (Theorem 6.3). As an example, we will give a 3-component Brunnian link $L=K_{1} \cup K_{2} \cup K_{3}$ such that $K_{1}$ is not null-homotopic in $S^{3} \backslash\left(L-K_{1}\right)$ and $K_{i}$ is null-homotopic in $S^{3} \backslash\left(L-K_{i}\right)(i=2,3)$ (Example 6.4). In particular, $L$ is link-homotopic to a trivial link. There is no such link with 2 components, i.e., if a 2-component link is link-homotopic to a trivial link, then it is self $\Delta$-equivalent to a Brunnian link $K_{1} \cup K_{2}$ such that $K_{i}$ is null-homotopic in $S^{3} \backslash K_{j}(\{i, j\}=\{1,2\})$.

For 2-component links, Theorem 1.2 can be generalized [22. Theorem 1.3 gives us the following corollary, which is a generalization of Theorem 1.2 for links with arbitrarily many components. This gives us a geometric characterization for links whose Milnor invariants with $r \leq 2$ vanish.

Corollary 1.5. A link $L$ is self $\Delta$-equivalent to a trivial link if and only if $\bar{\mu}_{L}(I)=0$ for any $I$ with $r(I) \leq 2$.

Remark 1.6. (1) This corollary gives an affirmative answer for an open question raised in 4 .

(2) For string links, Corollary 1.5 does not hold, i.e., there are 2-string links such that their Milnor invariants $\mu(I)$ with $r(I) \leq 2$ vanish and they are not self $\Delta$-equivalent to a trivial string link $[5]$.

(3) Since a $C_{k}$-move $(k \geq 3)$ is not an unknotting operation, it is impossible to generalize the corollary above. It is reasonable to consider the following question: If $\bar{\mu}_{L}(I)=0$ for any $I$ with $r(I) \leq k$, then is $L$ self $C_{k}$-equivalent to a completely split link? Fleming and the author gave a negative answer to the question [4]. In fact, there is a 2-component boundary link $L$ such that $L$ is not self $C_{3}$-equivalent to a split link. Note that all Milnor invariants of a boundary link vanish. We will give the definition of a boundary link after Remark 1.8.

By combining Lemma 3.2 ([19, Theorem 7]), Theorem 1.2 and Corollary 1.5 , we have the following corollary.

Corollary 1.7. Let $L$ be an $n$-component link and let $L(2)$ be a $2 n$-component link obtained from $L$ by replacing each component of $L$ with two zero framed parallel copies of it. Then $L$ is self $\Delta$-equivalent to a trivial link if and only if $L(2)$ is link-homotopic to a trivial link.

Remark 1.8. For an $n$-component link $L$, let $L(k)$ be a $k n$-component link obtained from $L$ by replacing each component of $L$ with $k$ zero framed parallel copies of it.

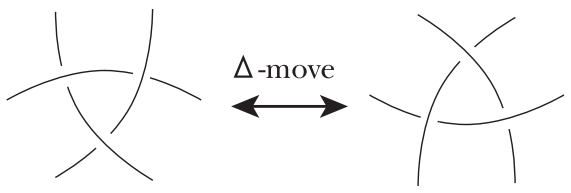

FiguRe 1.2 
In the proof of [4, Theorem 2.1], it is shown that if two links $L$ and $L^{\prime}$ are self $C_{k}$-equivalent, then $L(k)$ and $L^{\prime}(k)$ are link homotopic. So one might expect that if $L(2)$ and $L^{\prime}(2)$ are link homotopic, then $L$ and $L^{\prime}$ are self $\Delta$-equivalent. But this is not true. The reason is the following: There are 2-component links $L$ and $L^{\prime}$ such that they are concordant and are not self $\Delta$-equivalent 23], 24. The fact that $L$ and $L^{\prime}$ are concordant implies that $L(2)$ and $L^{\prime}(2)$ are concordant. Since link-concordance implies link-homotopy [6], 7], $L(2)$ and $L^{\prime}(2)$ are link-homotopic.

An $n$-component link $L=K_{1} \cup \cdots \cup K_{n}$ is called a boundary link if there is a disjoint union $X=F_{1} \cup \cdots \cup F_{n}$ of orientable surfaces such that $\partial X=L$ and $\partial F_{i}=K_{i}(i=1,2, \ldots, n)$. An $n$-component link $L$ is called a homology boundary link if $\pi_{1}\left(S^{3} \backslash L\right)$ admits an epimorphism from $\pi_{1}\left(S^{3} \backslash L\right)$ to a free group of rank $n$ 28. Every boundary link is a homology boundary link. T. Shibuya and the author showed that all boundary links are self $\Delta$-equivalent to trivial links [27]. In [25], Shibuya showed that all ribbon links are self $\Delta$-equivalent to trivial links.

Whether homology boundary links are self $\Delta$-equivalent to trivial links and whether slice links are self $\Delta$-equivalent to trivial links have remained as open questions. Since all Milnor invariants of homology boundary links vanish, and since Milnor invariants are concordance invariants [1, we have the following corollary, which gives affirmative answers for the open questions.

Corollary 1.9. If $L$ is concordant to a homology boundary link, then $L$ is self $\Delta$-equivalent to a trivial link.

\section{Claspers}

Let us briefly recall from [11] the basic notions of clasper theory for (string) links. In this paper, we essentially only need the notion of a $C_{k}$-tree. For a general definition of claspers, we refer the reader to [11.

Let $L$ be a link in $S^{3}$ (resp. a string link in $D^{2} \times I$ ). An embedded disk $F$ in $S^{3}$ (resp. $D^{2} \times I$ ) is called a tree clasper for $L$ if it satisfies the following (1), (2) and (3):

(1) $F$ is decomposed into disks and bands, called edges, each of which connects two distinct disks.

(2) The disks have either 1 or 3 incident edges, called leaves or nodes respectively.

(3) $L$ intersects $F$ transversely and the intersections are contained in the union of the interior of the leaves.

The degree of a tree clasper is the number of leaves minus 1. (In [11, a tree clasper and a leaf are called a strict tree clasper and a disk-leaf respectively.) A degree $k$ tree clasper is called a $C_{k}$-tree (or a $C_{k}$-clasper). A $C_{k}$-tree is simple if each leaf intersects $L$ at one point.

We will make use of the drawing convention for claspers of [11, Fig. 7], except for the following: $\oplus$ on an edge represents a positive half-twist. (This replaces the convention of a circled $S$ used in [11.)

Given a $C_{k}$-tree $T$ for a (string) link $L$, there is a procedure to construct a framed link $\gamma(T)$ in a regular neighborhood of $T$. Surgery along $T$ means surgery along $\gamma(T)$. Since there exists an orientation-preserving homeomorphism, fixing the boundary, from the regular neighborhood $N(T)$ of $T$ to the manifold $N(T)_{T}$ obtained from $N(T)$ by surgery along $T$, surgery along the $C_{k}$-tree $T$ can be regarded as a local move on $L$. We say that the resulting link $L_{T}$ is obtained from 


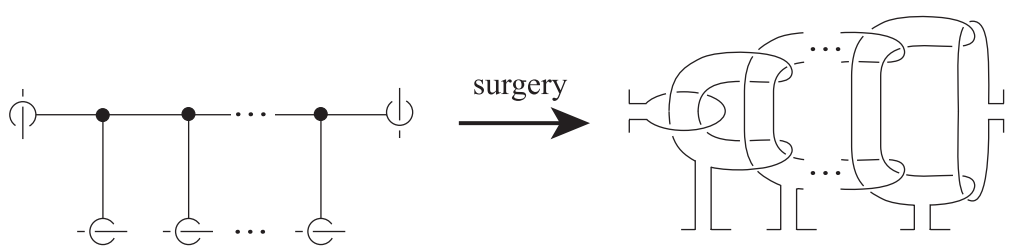

Figure 2.1. Surgery along a simple $C_{k}$-tree

$L$ by surgery along $T$. In particular, surgery along a simple $C_{k}$-tree, illustrated in Figure 2.1, is equivalent to band-summing a copy of the $(k+1)$-component Milnor link (see [18, Fig. 7]) and is equivalent to a $C_{k}$-move as defined in the introduction (Figure 1.1). Similarly, for a disjoint union of trees $T_{1} \cup \cdots \cup T_{m}$ for $L$, we can define $L_{T_{1} \cup \cdots \cup T_{m}}$ as a link obtained by surgery along $T_{1} \cup \cdots \cup T_{m}$. We often regard $L \cup T_{1} \cup \cdots \cup T_{m}$ as $L_{T_{1} \cup \cdots \cup T_{m}}$. A $C_{k}$-tree $T$ having the shape of the tree clasper in Figure 2.1 is called linear, and the left-most and right-most leaves of $T$ in Figure 2.1 are called the ends of $T$. Recall that a $C_{k}$-tree is an embedded disk. For a linear $C_{k}$-tree $T$, the ends of $T$ are uniquely determined.

It is known that the $C_{k}$-equivalence as defined in section 1 coincides with the equivalence relation on links generated by surgery along $C_{k}$-trees and ambient isotopy. Two (string) links $L$ and $L^{\prime}$ are $C_{k}$-equivalent if and only if there is a disjoint union of simple $C_{k}$-trees $G_{1} \cup \cdots \cup G_{m}$ such that $L^{\prime}$ is ambient isotopic to $L_{G_{1} \cup \cdots \cup G_{m}}$ [11, Theorem 3.17].

Let $L=K_{1} \cup \cdots \cup K_{n}$ be an $n$-component (string) link. A (simple) $C_{k}$-tree $T$ for $L$ is a (simple) $C_{k}^{a}$-tree (resp. $C_{k}^{d}$-tree, $C_{k}^{s}$-tree) if it satisfies the following:

(1) For each leaf $f$ of $T, f \cap L$ is contained in a single component of $L$, and

(2) $\left|\left\{i \mid T \cap K_{i} \neq \emptyset\right\}\right|=n$ (resp. $\left.=k+1,1\right)$.

Note that $n$ is the number of components of $L$ and that $k+1$ is the number of leaves of $T$. If $T$ is simple, $T$ always satisfies the condition (1). The superscript $a$ (resp. $d$ and $s$ ) stands for trees grasping all components (resp. distinct components and a single component). For an $n$-component link $L$, there exist $C_{k}^{a}$-trees for $L$ if and only if $n \leq k+1$, there exist $C_{k}^{d}$-trees for $L$ if and only if $n \geq k+1$, and there exist $C_{k}^{s}$-trees for $L$ for any $k \geq 1$. The $C_{k}^{*}$-equivalence $(*=a, d, s)$ is an equivalence relation on (string) links generated by surgery along $C_{k}^{*}$-trees and ambient isotopy. Note that $C_{k}^{s}$-equivalence is the same as self $C_{k^{-}}$-equivalence. In particular, $C_{k}^{s}$ equivalence coincides with the link-homotopy and self $\Delta$-equivalence for $k=1$ and $k=2$ respectively. For a simple $C_{k}$-tree $T$, the set $\left\{i \mid T \cap K_{i} \neq \emptyset\right\}$ is called the index of $T$, and is denoted by index $(T)$. Let $r_{i}(T)$ be the number of intersection points in $T \cap K_{i}(i=1, \ldots, n)$. The $\left(C_{l}^{s}+C_{k}^{*}\right)$-equivalence $\left(C_{k}^{*}=C_{k}, C_{k}^{a}\right.$, or $\left.C_{k}^{d}\right)$ is an equivalence relation on (string) links generated by surgery along $C_{l}^{s}$-trees or $C_{k}^{*}$-trees and ambient isotopy. By arguments similar to those in the proof of [11, Theorem 3.17], we have that two (string) links $L$ and $L^{\prime}$ are $\left(C_{l}^{s}+C_{k}^{*}\right)$-equivalent if and only if there is a disjoint union of simple $C_{l}^{s}$-trees or $C_{k}^{*}$-trees $T_{1} \cup \cdots \cup T_{m}$ such that $L^{\prime}$ is ambient isotopic to $L_{T_{1} \cup \cdots \cup T_{m}}$. We use the notation $L \stackrel{C_{k}^{*}}{\sim} L^{\prime}$ (resp. $\left.L \stackrel{C_{l}^{s}+C_{k}^{*}}{\sim} L^{\prime}\right)$ for $C_{k}^{*}$-equivalent (resp. $\left(C_{l}^{s}+C_{k}^{*}\right)$-equivalent) links $L$ and $L^{\prime}$.

Recall that a string link is a tangle without closed components (see 9] for a precise definition). The set of ambient isotopy classes of $n$-component string links 



FiguRe 2.2
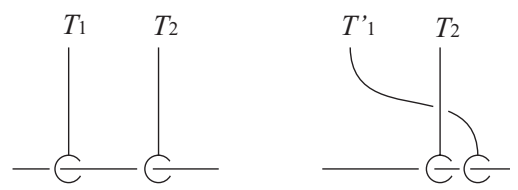

FiguRE 2.3. Sliding a leaf over another leaf

has a monoid structure with composition given by the stacking product, denoted by $*$, and with the trivial $n$-component string link $\mathbf{1}_{n}$ as the unit element.

In the following, we give several lemmas. The proofs of Lemmas 2.1, 2.2 and 2.4 are essentially given in [11] (see also section 1.4 in [15), and Lemma 2.5 is essentially shown in [8] (see also [3], 16]). These proofs in [3], 11, 15], [16] rely on Habiro's zip construction [11, section 3.3]. Lemmas 2.1, 2.2, 2.4 and 2.5] below follow from straightforward refinements of those proofs, keeping track of the index and $r_{j}$ of claspers.

Lemma 2.1 (cf. [11, Propositions 4.5, 4.6]). Let $T$ be a simple $C_{k}$-tree for an $n$-component (string) link $L$, and let $T^{\prime}$ (resp. $T^{\prime \prime}$, and $T^{\prime \prime \prime}$ ) be obtained from $T$ by changing a crossing of an edge and the ith component $K_{i}$ of $L$ (resp. an edge of $T$, and an edge of another simple tree clasper G) (see Figure 2.2). Then

(1) $L_{T} \stackrel{C_{k+1}}{\sim} L_{T^{\prime}}$, and the $C_{k+1}$-equivalence is realized by surgery along simple $C_{k+1}$-trees with index $\operatorname{index}(T) \cup\{i\}$ and $r_{j} \geq r_{j}(T)(j=1, \ldots, n)$.

(2) $L_{T} \stackrel{C_{k+1}}{\sim} L_{T^{\prime \prime}}, L_{T \cup G} \stackrel{C_{k+1}}{\sim} L_{T^{\prime \prime \prime} \cup G}$, and the $C_{k+1}$-equivalence is realized by surgery along simple $C_{k+1}$-trees with $r_{j} \geq r_{j}(T)(j=1, \ldots, n)$.

Proof. (1) In the proof of [11, Proposition 4.5], by using zip construction, it is shown that $L_{T^{\prime}}$ is obtained from $L_{T}$ by surgery along a simple $C_{k+1}$-tree $H$. It is not hard to see that index $(H)=\operatorname{index}(T) \cup\{i\}$ and $r_{j}(H) \geq r_{j}(T)(j=1, \ldots, n)$ under the zip construction.

(2) Since $T$ and $G$ are tree claspers, $T^{\prime \prime}$ and $T^{\prime \prime \prime}$ are obtained from $T$ by combining ambient isotopy and changing crossings of an edge of $T$ and components of $L$. Lemma 2.1 (1) completes the proof.

Lemma 2.2 (cf. [11, Propositions 4.4]). Let $T_{1}$ (resp. $T_{2}$ ) be a simple $C_{k}$-tree (resp. a simple tree clasper) for an $n$-component (string) link $L$, and let $T_{1}^{\prime}$ be obtained from $T_{1}$ by sliding a leaf of $T_{1}$ over a leaf of $T_{2}$ (see Figure 2.3). Then $L_{T_{1} \cup T_{2}} \stackrel{C_{k+1}}{\sim} L_{T_{1}^{\prime} \cup T_{2}}$, and the $C_{k+1}$-equivalence is realized by surgery along simple $C_{k+1}$-trees with $r_{j} \geq r_{j}\left(T_{1}\right)(j=1, \ldots, n)$.

Proof. Note that $L_{T_{1} \cup T_{2}}=\left(L_{T_{2}}\right)_{T_{1}}$ and $L_{T_{1}^{\prime} \cup T_{2}}=\left(L_{T_{2}}\right)_{T_{1}^{\prime}}$. Hence $T_{1}^{\prime}$ is obtained from $T_{1}$ by changing crossings of edges of $T_{1}$ and components of $L_{T_{2}}$. By 
Lemma 2.1 (1),

$$
L_{T_{1} \cup T_{2}}=\left(L_{T_{2}}\right)_{T_{1}} \stackrel{C_{k+1}}{\sim}\left(L_{T_{2}}\right)_{T_{1}^{\prime}}=L_{T_{1}^{\prime} \cup T_{2}},
$$

and the $C_{k+1}$-equivalence is realized by surgery along simple $C_{k+1}$-trees with $r_{j} \geq$ $r_{j}\left(T_{1}\right)(j=1, \ldots, n)$.

Remark 2.3. In Lemma 2.1 (2), it follows from [11, Proposition 4.6] that if $G$ is a simple $C_{l}$-tree, then $L_{T \cup G}$ is $C_{k+l+1}$-equivalent to $L_{T^{\prime \prime \prime} \cup G}$. It is not hard to see that the $C_{k+l+1}$-equivalence is realized by surgery along a simple $C_{k+l+1}$-tree with $r_{j}=r_{j}(T)+r_{j}(G)(j=1, \ldots, n)$. Similarly, in Lemma 2.2, it follows from 11. Proposition 4.4] that if $T_{2}$ is a simple $C_{l}$-tree, then $L_{T_{1} \cup T_{2}}$ is $C_{k+l}$-equivalent to $L_{T_{1}^{\prime} \cup T_{2}}$. Moreover, for each $j=1, \ldots, n$, the $C_{k+l}$-equivalence is realized by surgery along a simple $C_{k+l}$-tree with $r_{j}=r_{j}\left(T_{1}\right)+r_{j}\left(T_{2}\right)-1$ if the strand of $L$ in Figure 2.3 passing through the leaves of $T_{1}$ and $T_{2}$ is the $j$ th component of $L$, or with $r_{j}=r_{j}\left(T_{1}\right)+r_{j}\left(T_{2}\right)$ otherwise. Since we do not need such strong results to show our results in this paper, we only mention them as a remark but not as lemmas.

Lemma 2.4 (cf. [11, Claim on p.36]). Let $T$ be a simple $C_{k}$-tree for $\mathbf{1}_{n}$ and let $\bar{T}$ be a simple $C_{k}$-tree obtained from $T$ by adding a half-twist on an edge. Then $\left(\mathbf{1}_{n}\right)_{T} *\left(\mathbf{1}_{n}\right)_{\bar{T}} \stackrel{C_{k+1}}{\sim} \mathbf{1}_{n}$, and the $C_{k+1}$-equivalence is realized by surgery along simple $C_{k+1}$-trees with $r_{j} \geq r_{j}(T)(j=1, \ldots, n)$.

Proof. In the proof of [11, Claim on p.36], it is seen that there is a simple $C_{k}$-tree $\bar{T}^{\prime}$ for $\mathbf{1}_{n}$ such that $\left(\mathbf{1}_{n}\right)_{T \cup} \bar{T}^{\prime}$ is ambient isotopic to $\mathbf{1}_{n}$ and $\bar{T}^{\prime}$ is obtained from $\bar{T}$ by changing crossings of edges of $\bar{T}$ and components of $\mathbf{1}_{n}$. By Lemma 2.1 (1),

$$
\left(\mathbf{1}_{n}\right)_{T} *\left(\mathbf{1}_{n}\right)_{\bar{T}} \stackrel{C_{k+1}}{\sim}\left(\mathbf{1}_{n}\right)_{T} *\left(\mathbf{1}_{n}\right)_{\bar{T}^{\prime}}
$$

and by Lemmas $2.1(2)$ and 2.2 .

$$
\left(\mathbf{1}_{n}\right)_{T} *\left(\mathbf{1}_{n}\right)_{\bar{T}^{\prime}} \stackrel{C_{k+1}}{\sim}\left(\mathbf{1}_{n}\right)_{T \cup \bar{T}^{\prime}}=\mathbf{1}_{n} .
$$

Moreover the $C_{k+1}$-equivalence above is realized by surgery along simple $C_{k+1}$-trees with $r_{j} \geq r_{j}(\bar{T})\left(=r_{j}(T)\right)$ for each $j=1, \ldots, n$.

Lemma 2.5 (cf. 8, Theorem 6.7], [16, Lemma 2.9]). Consider simple $C_{k}$-trees $T_{I}, T_{H}$ and $T_{X}$ for $\mathbf{1}_{n}$ which differ only in a small ball as illustrated in Figure 2.4. Then $\left(\mathbf{1}_{n}\right)_{T_{I}} \stackrel{C_{k+1}}{\sim}\left(\mathbf{1}_{n}\right)_{T_{H}} *\left(\mathbf{1}_{n}\right)_{T_{X}}$, and the $C_{k+1}$-equivalence is realized by surgery along simple $C_{k+1}$-trees with $r_{j} \geq r_{j}\left(T_{I}\right)(j=1, \ldots, n)$.

Proof. By arguments similar to those in the proof of [16. Lemma 2.9], we have that there are two simple $C_{k}$-trees $T_{H}^{\prime}$ and $T_{X}^{\prime}$ for $\mathbf{1}_{n}$ such that $\left(\mathbf{1}_{n}\right)_{T_{H}^{\prime} \cup T_{X}^{\prime}}$ is ambient isotopic to $\left(\mathbf{1}_{n}\right)_{T_{I}}$, and $T_{H}^{\prime}$ (resp. $T_{X}^{\prime}$ ) is obtained from $T_{H}$ (resp. $T_{X}$ ) by changing

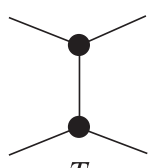

TI

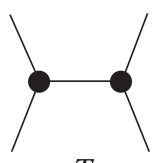

$T_{H}$

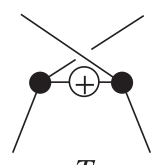

$T X$

Figure 2.4. The IHX relation for $C_{k}$-trees 

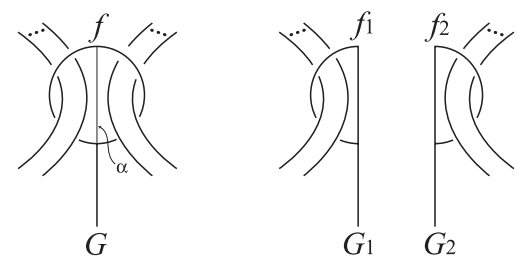

Figure 2.5. Splitting a leaf. The bunch of parallel strands passing through $f$ is a bunch of strands of components of $\mathbf{1}_{n}$

crossings of edges of $T_{H}$ (resp. $T_{X}$ ) and components of $\mathbf{1}_{n}$. By Lemmas 2.1 and 2.2 .

$$
\left(\mathbf{1}_{n}\right)_{T_{H}} *\left(\mathbf{1}_{n}\right)_{T_{X}} \stackrel{C_{k+1}}{\sim}\left(\mathbf{1}_{n}\right)_{T_{H}^{\prime}} *\left(\mathbf{1}_{n}\right)_{T_{X}^{\prime}} \stackrel{C_{k+1}}{\sim}\left(\mathbf{1}_{n}\right)_{T_{H}^{\prime} \cup T_{X}^{\prime}}=\left(\mathbf{1}_{n}\right)_{T_{I}},
$$

and the $C_{k+1}$-equivalence is realized by surgery along simple $C_{k+1}$-trees with $r_{j} \geq$ $r_{j}\left(T_{I}\right)(j=1, \ldots, n)$.

By combining the proof of [11, Claim on p.26] and Lemmas 2.1 and 2.2 (or 11, Propositions 4.4, 4.5 and 4.6]), we have the following.

Lemma 2.6 (cf. 11, Claim on p.26]). Let $G$ be a $C_{k}$-tree for $\mathbf{1}_{n}$. Let $f_{1}$ and $f_{2}$ be two disks obtained by splitting a leaf $f$ of $G$ along an arc $\alpha$ as shown in Figure 2.5 (i.e., $f=f_{1} \cup f_{2}$ and $f_{1} \cap f_{2}=\alpha$ ). Then, $\left(\mathbf{1}_{n}\right)_{G} \stackrel{C_{k+1}}{\sim}\left(\mathbf{1}_{n}\right)_{G_{1}} *\left(\mathbf{1}_{n}\right)_{G_{2}}$, where $G_{i}$ denotes the $C_{k}$-tree for $\mathbf{1}_{n}$ obtained from $G$ by replacing $f$ with $f_{i}(i=1,2)$.

Proof. In the proof of [11, Claim on p.26], it is shown that there is a simple $C_{k}$-tree $G_{2}^{\prime}$ for $\mathbf{1}_{n}$ such that $\left(\mathbf{1}_{n}\right)_{G_{1} \cup G_{2}^{\prime}}$ is ambient isotopic to $\left(\mathbf{1}_{n}\right)_{G}$ and $G_{2}^{\prime}$ is obtained from $G_{2}$ by changing crossings of edges of $G_{2}$ and components of $\mathbf{1}_{n}$. By Lemmas 2.1 (1) and 2.2, we have

$$
\left(\mathbf{1}_{n}\right)_{G_{1}} *\left(\mathbf{1}_{n}\right)_{G_{2}} \stackrel{C_{k+1}}{\sim}\left(\mathbf{1}_{n}\right)_{G_{1}} *\left(\mathbf{1}_{n}\right)_{G_{2}^{\prime}} \stackrel{C_{k+1}}{\sim}\left(\mathbf{1}_{n}\right)_{G_{1} \cup G_{2}^{\prime}}=\left(\mathbf{1}_{n}\right)_{G} .
$$

This completes the proof.

Remark 2.7. In the proof of Lemma 2.6, we note that the $C_{k+1}$-equivalence is realized by surgery along a simple $C_{k+1}$-tree with $r_{j} \geq r_{j}(G)(j=1, \ldots, n)$.

An $n$-component (string) link $L$ is Brunnian if every proper sublink of $L$ is trivial. In particular, any trivial (string) link is Brunnian. The $n$-component Brunnian (string) links are characterized by $C_{n-1}^{a}$-equivalence as follows.

Proposition $2.8([12,20])$. Let $L$ be an $n$-component (string) link in $S^{3}$. Then $L$ is Brunnian if and only if $L$ is obtained from a trivial (string) link by surgery along simple $C_{n-1}^{a}$-trees.

By arguments similar to those in the proof of Theorem 1.2 in 20, we have the following lemma. In 20, the authors proved it with using 'band description' defined by K. Taniyama and the author [29. Here we give a proof with using clasper.

Lemma 2.9 (cf. 20, Theorem 1.2]). Let $L$ be an n-component Brunnian link in $S^{3}$. If $L$ is obtained from a trivial link $O$ by surgery along $C_{1}^{s}$-trees with index $\{i\}$, then $L$ is obtained from $O$ by surgery along simple $C_{n}^{a}$-trees with $r_{i}=2$. 
Proof. Set $O=O_{1} \cup \cdots \cup O_{n}$. It is enough to consider the case when $i=1$. There is a disjoint union $F_{1}$ of simple $C_{1}^{s}$-trees with index $\{1\}$ such that $L=O_{F_{1}}$. Note that $r_{1}=2$ for all $C_{1}^{s}$-trees in $F_{1}$. Hence $L$ can be regarded as a union $\left(O_{1}\right)_{F_{1}} \cup O_{2} \cup \cdots \cup O_{n}$.

Since $L$ is Brunnian, $L \backslash O_{2}$ is the trivial link. This implies that a split sum of $L \backslash O_{2}$ and $O_{2}$ is trivial. Hence $L$ can be deformed into the trivial link by crossing changes between $O_{2}$ and edges of $C_{1}^{s}$-trees of $F_{1}$; here $O \cup F_{1}$ is regarded as $L=O_{F_{1}}$. By Lemma 2.1 (1), we have that $L$ is obtained from $O$ by surgery along a disjoint union $F_{2}$ of simple $C_{2}$-trees with index $\{1,2\}$ and $r_{1}=2$. So we have $L=O_{F_{2}}\left(=\left(O_{1} \cup O_{2}\right)_{F_{2}} \cup O_{3} \cup \cdots \cup O_{n}\right)$.

Since $L \backslash O_{3}$ is trivial, $L$ can be deformed into the trivial link by crossing changes between $O_{3}$ and edges of $C_{2}$-trees in $F_{2}$. By Lemma 2.1 (1), there is a disjoint union $F_{3}$ of simple $C_{3}$-trees with index $\{1,2,3\}$ and $r_{1}=2$ such that $L=$ $O_{F_{3}}\left(=\left(O_{1} \cup O_{2} \cup O_{3}\right)_{F_{3}} \cup O_{4} \cup \cdots \cup O_{n}\right)$.

Repeating this step, we have that there is a disjoint union $F_{n}$ of simple $C_{n}$-trees with index $\{1, \ldots, n\}$ and $r_{1}=2$ such that $L=O_{F_{n}}$. This completes the proof.

By arguments similar to those in the proof of [4, Proposition 3.1], we have

Proposition 2.10 (cf. [4, Proposition 3.1]). If an n-component (string) link $L^{\prime}$ is obtained from $L$ by surgery along a simple tree clasper $T$, then for any integers $p_{i}\left(0 \leq p_{i} \leq r_{i}(T), i=1, \ldots, n\right)$ with $k=p_{1}+\cdots+p_{n} \geq 2, L^{\prime}$ is obtained from $L$ by surgery along simple $C_{k-1}$-trees with $r_{i}=p_{i}(i=1, \ldots, n)$.

A simple $C_{k^{-}}$-tree $T$ for an $n$-component link is a $C_{k}^{(l)}$-tree if $\max \left\{r_{j}(T) \mid j=\right.$ $1, \ldots, n\} \geq l$. Two links $L$ and $L^{\prime}$ are $C_{k}^{(l)}$-equivalent if $L$ is obtained from $L^{\prime}$ by ambient isotopy and surgery along simple $C_{k}^{(l)}$-trees. The following proposition is a corollary of Proposition 2.10.

Proposition 2.11 (cf. [4, Proposition 3.1]). If two (string) links are $C_{m}^{(k+1)}$ equivalent, then they are self $C_{k}$-equivalent. Moreover, for some $i$, if the $C_{m}^{(k+1)}$ equivalence is realized by surgery along simple $C_{m}^{(k+1)}$-trees with $r_{i} \geq k+1$, then the self $C_{k}$-equivalence is realized by surgery along simple $C_{k}^{s}$-trees with index $\{i\}$.

\section{MiLnor inVARIAnts}

Milnor defined in [18, 19] a family of invariants of oriented, ordered links in $S^{3}$, known as Milnor's $\bar{\mu}$-invariants.

Given an $n$-component link $L=K_{1} \cup \cdots \cup K_{n}$ in $S^{3}$, denote by $G$ the fundamental group of $S^{3} \backslash L$, and by $G_{q}$ the $q$ th subgroup of the lower central series of $G$. We have a presentation of $G / G_{q}$ with $n$ generators, given by a meridian $m_{i}$ of each component $K_{i}$. So, for $1 \leq i \leq n$, the longitude $l_{i}$ of the $i$ th component of $L$ is expressed modulo $G_{q}$ as a word in the $m_{i}$ 's (abusing notation, we still denote this word by $l_{i}$ ).

The Magnus expansion $E\left(l_{i}\right)$ of $l_{i}$ is the formal power series in non-commuting variables $X_{1}, \ldots, X_{n}$ obtained by substituting $1+X_{j}$ for $m_{j}$ and $1-X_{j}+X_{j}^{2}-X_{j}^{3}+\cdots$ for $m_{j}^{-1}, 1 \leq j \leq n$.

Let $I=i_{1} i_{2} \ldots i_{k-1} j(k \leq q)$ be a multi-index among $\{1, \ldots, n\}$. Denote by $\mu_{L}(I)$ the coefficient of $X_{i_{1}} \cdots X_{i_{k-1}}$ in the Magnus expansion $E\left(l_{j}\right)$. The Milnor invariant $\bar{\mu}_{L}(I)$ is the residue class of $\mu_{L}(I)$ modulo the greatest common divisor of 


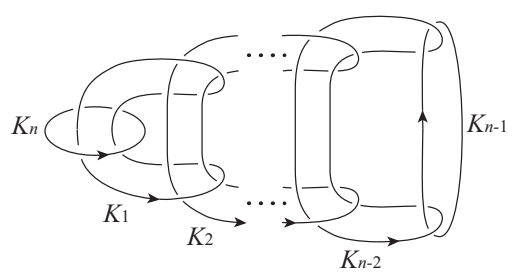

Figure 3.1. The Milnor link $M_{n}$

all Milnor invariants $\mu_{L}(J)$ such that $J$ is obtained from $I$ by removing at least one index, and permutating the remaining indices cyclicly. As we mentioned in section $1,|I|=k$ is called the length of the Milnor invariant $\bar{\mu}_{L}(I)$. It is known that $\bar{\mu}_{L}(I)$ are concordance invariants [1.

The indeterminacy comes from the choice of the meridians $m_{i}$. Equivalently, it comes from the indeterminacy of representing the link as the closure of a string link 9. Indeed, $\mu(I)$ is a well-defined invariant for string links.

The following 4 lemmas play an important role in calculating Milnor invariants.

Lemma 3.1 ([18, section 5]). Let $M_{n}=K_{1} \cup \cdots \cup K_{n}$ be the $n$-component Milnor link as illustrated in Figure 3.1. Then the Milnor invariants of length $\leq n-1$ vanish, and

$$
\bar{\mu}_{M_{n}}\left(i_{1} i_{2} \ldots i_{n-2} n-1 n\right)= \begin{cases}1 & \text { if } i_{1} i_{2} \ldots i_{n-2}=12 \ldots n-2 \\ 0 & \text { otherwise }\end{cases}
$$

Lemma 3.2 ([19, Theorem 7]). Let $L^{\prime}$ be a link obtained from a link $L$ by taking the appropriate number of zero framed parallels of the components of L. Suppose the ith component of $L^{\prime}$ corresponds to the $h(i)$ th component of $L$. Then

$$
\bar{\mu}_{L^{\prime}}\left(i_{1} i_{2} \ldots i_{m}\right)=\bar{\mu}_{L}\left(h\left(i_{1}\right) h\left(i_{2}\right) \ldots h\left(i_{m}\right)\right) .
$$

Lemma 3.3 ([17, Lemma 3.3]). Let $L$ and $L^{\prime}$ be $n$-component string links such that all Milnor invariants of $L$ (resp. $\left.L^{\prime}\right)$ of length $\leq m\left(\right.$ resp. $\left.\leq m^{\prime}\right)$ vanish. Then $\mu_{L * L^{\prime}}(I)=\mu_{L}(I)+\mu_{L^{\prime}}(I)$ for all $I$ of length $\leq m+m^{\prime}$.

Lemma 3.4 ([11, Theorem 7.2]). The Milnor invariants of length $\leq k$ for (string) links are invariants of $C_{k}$-equivalence.

\section{LINK-HOMOTOPY OF STRING LINKS}

Let $\pi:\{1, \ldots, k\} \longrightarrow\{1, \ldots, n\}(k \leq n)$ be an injection such that $\pi(i)<\pi(k-1)<$ $\pi(k)(i \in\{1, \ldots, k-2\})$, and let $\mathcal{F}_{k}$ be the set of such injections. For $\pi \in \mathcal{F}_{k}$, let $T_{\pi}$ and $\bar{T}_{\pi}$ be simple linear $C_{k-1}^{d}$-trees as illustrated in Figure 4.1, and set $V_{\pi}=\left(\mathbf{1}_{n}\right)_{T_{\pi}}$ and $V_{\pi}^{-1}=\left(\mathbf{1}_{n}\right)_{\bar{T}_{\pi}}$. Here, Figure 4.1 gives the images of homeomorphisms from the neighborhoods of $T_{\pi}$ and $\bar{T}_{\pi}$ to the 3-ball. Although $V_{\pi}$ and $V_{\pi}^{-1}$ are not unique up to ambient isotopy, by Lemmas 2.1 and 2.4 they are unique up to $C_{k^{-}}$ equivalence. So, for any $\pi \in \mathcal{F}_{k}$, we may choose $V_{\pi}$ and $V_{\pi}^{-1}$ uniquely up to $C_{k}$-equivalence. In particular, we may choose $V_{\pi}$ so that $\operatorname{cl}\left(V_{\pi}\right)$ is the Milnor link $M_{\pi}=K_{\pi(1)} \cup \cdots \cup K_{\pi(k)}$ as illustrated in Figure 4.2 (cf. Figure 2.1).

For $\pi \in \mathcal{F}_{k}$, set

$$
\mu_{\pi}(L)=\mu_{L}(\pi(1) \pi(2) \ldots \pi(k)) .
$$

By Lemma 3.1, we have the following lemma. 



FIgURE 4.1. $\pi(i)$ means the $\pi(i)$ th component of $\mathbf{1}_{n}(i=1, \ldots, k)$

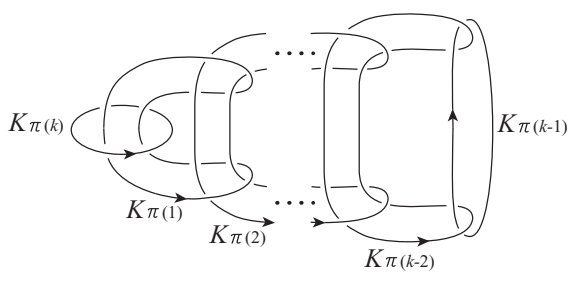

FIgURE 4.2. Milnor link $M_{\pi}=K_{\pi(1)} \cup \cdots \cup K_{\pi(k)}$

Lemma 4.1. For any $\pi, \pi^{\prime} \in \mathcal{F}_{k}$,

$$
\mu_{\pi}\left(V_{\pi^{\prime}}\right)=\left\{\begin{array}{ll}
1 & \text { if } \pi=\pi^{\prime} \\
0 & \text { if } \pi \neq \pi^{\prime}
\end{array},\right.
$$

and the Milnor invariants of $V_{\pi^{\prime}}$ of length $\leq k-1$ vanish.

Lemma 4.2. Let $T$ be a simple $C_{k-1}^{d}$-tree (resp. $C_{n-1}^{a}$-tree) for an $n$-component string link $L(k \leq n)$. Then $L_{T}$ is $C_{k}$-equivalent (resp. $C_{n}^{a}$-equivalent) to $L * L^{\prime}$, where

$$
L^{\prime}=\prod_{\pi \in \mathcal{F}_{k}} V_{\pi}^{\mu_{\pi}\left(L_{T}\right)-\mu_{\pi}(L)}\left(\text { resp. }=\prod_{\pi \in \mathcal{F}_{n}} V_{\pi}^{\mu_{\pi}\left(L_{T}\right)-\mu_{\pi}(L)}\right) .
$$

Proof. Suppose that $T$ is a simple $C_{k-1}^{d}$-tree. By Lemma2.1 $L_{T}$ is $C_{k}$-equivalent to $L *\left(\mathbf{1}_{n}\right)_{T^{\prime}}$, where $T^{\prime}$ is a simple $C_{k-1}^{d}$-tree. Set index $\left(T^{\prime}\right)=\left\{i_{1}, \ldots, i_{k}\right\}\left(i_{j}<i_{j+1}\right)$. Consider induction on the length of the path connecting the two leaves grasping the $i_{k-1}$ th and $i_{k}$ th components of $\mathbf{1}_{n}$, and apply Lemmas 2.5 and 2.4 we have that $\left(\mathbf{1}_{n}\right)_{T^{\prime}}$ is $C_{k}$-equivalent to a string link which is obtained from $\mathbf{1}_{n}$ by surgery along simple linear $C_{k-1}^{d}$-trees whose ends grasp the $i_{k-1}$ th and $i_{k}$ th components of $\mathbf{1}_{n}$. By Lemmas 2.1, 2.2 and 2.4, we have that

$$
\left(\mathbf{1}_{n}\right)_{T^{\prime}} \stackrel{C_{k}}{\sim} L^{\prime \prime}=\prod_{\pi \in \mathcal{F}_{k}} V_{\pi}^{x_{\pi}} .
$$

By Lemmas 3.43 .3 and 4.1 ,

$$
\begin{aligned}
\mu_{\pi^{\prime}}\left(L_{T}\right)=\mu_{\pi^{\prime}}\left(L * L^{\prime \prime}\right) & =\mu_{\pi^{\prime}}(L)+\mu_{\pi^{\prime}}\left(L^{\prime \prime}\right) \\
& =\mu_{\pi^{\prime}}(L)+\sum_{\pi \in \mathcal{F}_{k}} x_{\pi} \mu_{\pi^{\prime}}\left(V_{\pi}\right)=\mu_{\pi^{\prime}}(L)+x_{\pi^{\prime}} .
\end{aligned}
$$

Suppose that $T$ is a simple $C_{n-1}^{a}$-tree. Since a $C_{n-1}^{a}$-tree is a $C_{n-1}^{d}$-tree, we have already shown that

$$
L_{T} \stackrel{C_{n}}{\sim} L * \prod_{\pi \in \mathcal{F}_{n}} V_{\pi}^{\mu_{\pi}\left(L_{T}\right)-\mu_{\pi}(L)} .
$$


Lemmas 2.1, 2.2, 2.4 and 2.5 imply that the $C_{n}$-equivalence is realized by surgery along $C_{n}^{a}$-trees.

The following theorem gives representatives, which depend only on Milnor invariants, for the link-homotopy classes.

Theorem 4.3. Let $L$ be an $n$-component string link. Then $L$ is link-homotopic to $L_{1} * L_{2} * \cdots * L_{n-1}$, where

$$
\begin{gathered}
L_{i}=\prod_{\pi \in \mathcal{F}_{i+1}} V_{\pi}^{x_{\pi}}, \\
x_{\pi}= \begin{cases}\mu_{L}(\pi(1) \pi(2)) & \text { if } i=1, \\
\mu_{L}(\pi(1) \ldots \pi(i+1))-\mu_{L_{1} * \cdots * L_{i-1}}(\pi(1) \ldots \pi(i+1)) & \text { if } i \geq 2 . \\
\left(=\mu_{L_{i}}(\pi(1) \ldots \pi(i+1))\right) & \end{cases}
\end{gathered}
$$

Remark 4.4. The presentation $L_{1} * L_{2} * \cdots * L_{n-1}$ of $L$ depends on the choice of order on the elements in $\mathcal{F}_{i}(i=2, \ldots, n)$. If we put $\mathcal{F}_{2} \cup \cdots \cup \mathcal{F}_{n}=\left\{\pi_{1}, \ldots, \pi_{q}\right\}$ so that for $i<j$, any element in $\mathcal{F}_{i}$ appears before the elements in $\mathcal{F}_{j}$, then by Theorem 4.3 and Lemmas 3.3 and $4.1, L$ is link-homotopic to

$$
V_{\pi_{1}}^{x_{\pi_{1}}} * \cdots * V_{\pi_{q}}^{x_{\pi_{q}}}\left(x_{\pi_{k}}=\mu_{\pi_{k}}(L)-\mu_{\pi_{k}}\left(\prod_{i=1}^{k-1} V_{\pi_{i}}^{x_{\pi_{i}}}\right)\right) .
$$

Note that the representation is unique up to link-homotopy.

Proof. Since the $C_{1}$-move is the crossing change, $L$ is $C_{1}$-equivalent to the trivial string link $\mathbf{1}_{n}$. So $L$ is obtained from $\mathbf{1}_{n}$ by surgery along simple $C_{1}$-trees.

Note that a simple $C_{1}$-tree is either a simple $C_{1}^{s}$-tree or a simple $C_{1}^{d}$-tree, and that $C_{1}^{s}$-equivalence preserves the value of $\mu(I)$ for any $I$ with $r(I)=1$. Since $L$ is $C_{1}^{s}$-equivalent to a link which is obtained from $\mathbf{1}_{n}$ by surgery along $C_{1}^{d}$-trees, by Lemmas 4.2, 2.1, 2.2 and 2.4,

$$
L \stackrel{C_{1}^{s}+C_{2}}{\sim} \prod_{\pi \in \mathcal{F}_{2}} V_{\pi}^{\mu_{\pi}(L)}\left(=L_{1}\right) .
$$

A $C_{k}$-tree $(k \geq 2)$ is either a $C_{k}^{(2)}$-tree or a $C_{k}^{d}$-tree, and $C_{k}^{(2)}$-equivalence implies $C_{1}^{s}$-equivalence (Proposition 2.11). It follows that $\left(C_{1}^{s}+C_{k}\right)$-equivalence implies $\left(C_{1}^{s}+C_{k}^{d}\right)$-equivalence. So $L$ is obtained from $L_{1}$ by surgery along simple $C_{1}^{s}$-trees and $C_{2}^{d}$-trees.

By Lemmas 4.2, 2.1, 2.2 and 2.4,

$$
L \stackrel{C_{1}^{s}+C_{3}}{\sim} L_{1} * \prod_{\pi \in \mathcal{F}_{3}} V_{\pi}^{\mu_{\pi}(L)-\mu_{\pi}\left(L_{1}\right)}\left(=L_{1} * L_{2}\right) .
$$

Therefore $L$ and $L_{1} * L_{2}$ are $\left(C_{1}^{s}+C_{3}^{d}\right)$-equivalent.

Repeating this process, we have that

$$
L \stackrel{C_{1}^{s}+C_{n}}{\sim} L_{1} * L_{2} * \cdots * L_{n-1} .
$$

Since any simple $C_{n}$-tree for an $n$-component string link is a $C_{n}^{(2)}$-tree, $\left(C_{1}^{s}+C_{n}\right)$ equivalence implies $C_{1}^{s}$-equivalence, i.e., link-homotopy. 

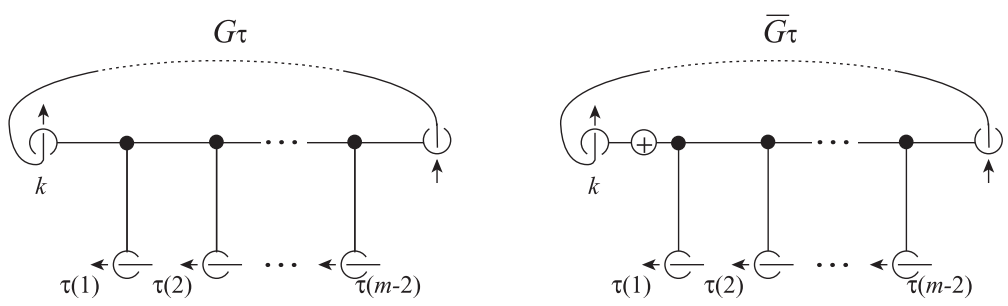

Figure 5.1. $\tau(i)(i=1, \ldots, m-2)$ and $k$ mean the $\tau(i)$ th and $k$ th components of $\mathbf{1}_{n}$, respectively

By Theorem 4.3, we have the following corollary.

Corollary 4.5. For a natural number $k \leq n$, two $n$-component string links $L$ and $L^{\prime}$ are $\left(C_{1}^{s}+C_{k}\right)$-equivalent if and only if $\mu_{L}(I)=\mu_{L^{\prime}}(I)$ for any $I$ with $r(I)=1$ and $|I| \leq k$.

Proof. The 'only if' part follows from Lemma 3.4. Now we will prove the 'if' part.

By Theorem 4.3, $L$ and $L^{\prime}$ are link-homotopic to $L_{1} * L_{2} * \cdots * L_{n-1}$ and $L_{1}^{\prime} * L_{2}^{\prime} *$ $\cdots * L_{n-1}^{\prime}$ respectively. Note that both $L_{i}$ and $L_{i}^{\prime}$ are $C_{i}$-equivalent to $\mathbf{1}_{n}$. So $L$ and $L^{\prime}$ are $\left(C_{1}^{s}+C_{k}\right)$-equivalent to $L_{1} * L_{2} * \cdots * L_{k-1}$ and $L_{1}^{\prime} * L_{2}^{\prime} * \cdots * L_{k-1}^{\prime}$ respectively. Since $\mu_{L}(I)=\mu_{L^{\prime}}(I)$ for any $I$ with $r(I)=1$ and $|I| \leq k, L_{i}=L_{i}^{\prime}(i=1, \ldots, k-1)$. This completes the proof.

Theorem 1.1 follows directly from Corollary 4.5.

Proof of Theorem 1.1. It is enough to show the 'if' part. Since a $C_{n}$-move for an $n$-component string link is a $C_{n}^{(2)}$-move, by Proposition 2.11, $C_{n}$-equivalence implies $C_{1}^{s}$-equivalence. Hence $\left(C_{1}^{s}+C_{n}\right)$-equivalence implies link-homotopy. By Corollary [.5] $L$ and $L^{\prime}$ are link-homotopic.

Remark 4.6. Let $L$ be an $n$-component link in $S^{3}$. Denote by $\mathcal{L}(L)$ the set of all $n$-component string links $l$ such that $\operatorname{cl}(l)=L$. Put $\mathcal{F}_{2} \cup \cdots \cup \mathcal{F}_{n}=\left\{\pi_{1}, \ldots, \pi_{q}\right\}$ so that any element in $\mathcal{F}_{i}$ appears before the elements in $\mathcal{F}_{j}(2 \leq i<j \leq n)$ and fix this total order on $\mathcal{F}_{2} \cup \cdots \cup \mathcal{F}_{n}$. Then, by Remark 4.4, each $l$ in $\mathcal{L}(L)$ is linkhomotopic to $V_{\pi_{1}}^{x_{\pi_{1}}} * \cdots * V_{\pi_{q}}^{x_{\pi_{q}}}\left(x_{\pi_{k}}=\mu_{\pi_{k}}(l)-\mu_{\pi_{k}}\left(\prod_{i=1}^{k-1} V_{\pi_{i}}^{x_{\pi_{i}}}\right)\right)$, which is the unique representation up to link-homotopy. We define a vector $v_{l}$ as $v_{l}=\left(x_{\pi_{1}}, \ldots, x_{\pi_{q}}\right)$, and set $\mathcal{V}_{L}=\left\{v_{l} \mid l \in \mathcal{L}(L)\right\}$. By the uniqueness of the presentation for $l$, we have the following: Two $n$-component links $L$ and $L^{\prime}$ in $S^{3}$ are link-homotopic if and only if $\mathcal{V}_{L} \cap \mathcal{V}_{L^{\prime}} \neq \emptyset$.

\section{Self $\Delta$-Equivalence of Brunnian links}

Let $n$ and $m$ be integers $(2 \leq n<m \leq 2 n)$. Given $k \in\{1, \ldots, n\}$, consider a surjection $\tau$ from $\{1, \ldots, m-2\}$ to $\{1, \ldots, n\} \backslash\{k\}$. Let $G_{\tau}$ and $\bar{G}_{\tau}$ be the simple linear $C_{m-1}^{a}$-trees illustrated in Figure 5.1 and set $V_{\tau}=\left(\mathbf{1}_{n}\right)_{G_{\tau}}$ and $V_{\tau}^{-1}=\left(\mathbf{1}_{n}\right)_{\bar{G}_{\tau}}$. Here, Figure 5.1 are the images of homeomorphisms from the neighborhoods of $G_{\tau}$ and $\bar{G}_{\tau}$ to the 3-ball. Although $V_{\tau}$ and $V_{\tau}^{-1}$ are not unique up to ambient isotopy, by Lemmas 2.1 and 2.4, their closures are unique up to $C_{m}^{a}$-equivalence. For each $\tau$, we choose $V_{\tau}$ and $V_{\tau}^{-1}$ and fix them.

Set

$$
\mu_{\tau}(L)=\mu_{L}(\tau(1) \ldots \tau(m-2) k k) .
$$


Recall that $2 \leq n<m \leq 2 n$. Let $\mathcal{B}_{m}(k)$ be the set of all surjections $\tau$ from $\{1, \ldots, m-2\}$ to $\{1, \ldots, n\} \backslash\{k\}$ such that $\left|\tau^{-1}(i)\right| \leq 2(i=1, \ldots, n)$ and $\left|\tau^{-1}(j)\right|=1$ (if $j>k$ ), and let $\rho_{m}$ be a surjection from $\{1, \ldots, m-2\}$ to itself defined by $\rho_{m}(i)=m-1-i$. Note that the definition of $\mathcal{B}_{m}(k)$ implies that $\mathcal{B}_{m}(k)=\emptyset$ if $k<m-n$. So we may assume that $k \geq m-n$.

If $m \leq 2 n-2$, then for any $\tau \in \mathcal{B}_{m}(k),\{i \mid \tau(i) \neq \tau(m-1-i)\} \neq \emptyset$. We set

$$
\mathcal{P}_{m}(k)=\left\{\begin{array}{l|l}
\tau \in \mathcal{B}_{m}(k) & \begin{array}{l}
\tau(p)<\tau(m-1-p) \\
\text { for } p=\min \{i \mid \tau(i) \neq \tau(m-1-i)\}
\end{array}
\end{array}\right\} .
$$

See Figure 5.2 (a) and (b): $\tau_{1}$ is an element in $\mathcal{P}_{6}(2)\left(=\mathcal{P}_{2 n-2}(m-n)\right)$, and $\tau_{1} \rho_{6}$ is an element in $\mathcal{B}_{6}(2) \backslash \mathcal{P}_{6}(2)$.

If $m=2 n-1$, then $k=n, n-1$ and there is $\tau \in \mathcal{B}_{2 n-1}(n) \cup \mathcal{B}_{2 n-1}(n-1)$ such that $\tau(i)=\tau(2 n-2-i)(i=1, \ldots, n-2)$ and $\left|\tau^{-1}(\tau(n-1))\right|=1$. For $k=n, n-1$, set

$$
\mathcal{R}_{2 n-1}(k)=\left\{\begin{array}{l|l}
\tau \in \mathcal{B}_{2 n-1}(k) \mid \begin{array}{l}
\tau(i)=\tau(2 n-2-i)(i=1, \ldots, n-2), \\
\left|\tau^{-1}(\tau(n-1))\right|=1
\end{array}
\end{array}\right\},
$$

and set

$$
\mathcal{P}_{2 n-1}(k)=\left\{\begin{array}{l|l}
\tau \in \mathcal{B}_{2 n-1}(k) \backslash \mathcal{R}_{2 n-1}(k) & \begin{array}{l}
\tau(p)<\tau(2 n-2-p) \\
\text { for } p=\min \{i \mid \tau(i) \neq \tau(2 n-2-i)\}
\end{array}
\end{array}\right\} .
$$

Note that if $\tau \in \mathcal{R}_{2 n-1}(n-1)$, then $\tau(n-1)=n$. See Figure 5.2 (c): $\tau_{2}$ is an element in $\mathcal{R}_{7}(3)\left(=\mathcal{R}_{2 n-1}(n-1)\right)$.

If $m=2 n$, then $k=n$ and there is $\tau \in \mathcal{B}_{2 n}(n)$ such that $\tau(i)=\tau(2 n-1-i)$ $(i=1, \ldots, n-1)$. Set

$$
\mathcal{R}_{2 n}(n)=\left\{\tau \in \mathcal{B}_{2 n}(n) \mid \tau(i)=\tau(2 n-1-i)(i=1, \ldots, n-1)\right\},
$$

and set

$$
\mathcal{P}_{2 n}(n)=\left\{\begin{array}{l|l}
\tau \in \mathcal{B}_{2 n}(n) \backslash \mathcal{R}_{2 n}(n) \mid \begin{array}{l}
\tau(p)<\tau(2 n-1-p) \\
\text { for } p=\min \{i \mid \tau(i) \neq \tau(2 n-1-i)\}
\end{array}
\end{array}\right\} .
$$

See Figure 5.2 (d): $\tau_{3}$ is an element in $\mathcal{R}_{8}(4)\left(=\mathcal{R}_{2 n}(n)\right)$.

We note that if $\tau \in \mathcal{R}_{m}(k)$, then $\tau \rho_{m} \in \mathcal{R}_{m}(k)$ (i.e., $\tau$ has 'symmetry'); if $\tau \in \mathcal{P}_{m}(k)$, then $\tau \rho_{m} \notin \mathcal{P}_{m}(k)$, and

$$
\mathcal{B}_{m}(k)=\mathcal{P}_{m}(k) \cup \mathcal{R}_{m}(k) \cup\left\{\tau \rho_{m} \mid \tau \in \mathcal{P}_{m}(k)\right\} .
$$

For any $\varphi \in \mathcal{B}_{m}(k), V_{\varphi}$ is $C_{m-1}$-equivalent to $\mathbf{1}_{n}$. By Lemma 3.4 $\mu_{V_{\varphi}}(I)=0$ for any $I$ with $|I| \leq m-1$.

By arguments similar to those in the proof of [17, Proposition 5.1], we have the following lemma.

Lemma 5.1. (1) If $\tau \in \mathcal{P}_{m}(k)$, then for an $n$-component string link $L$,

$$
\operatorname{cl}\left(L * V_{\tau \rho_{m}}\right) \stackrel{C_{m}^{a}}{\sim} \begin{cases}\operatorname{cl}\left(L * V_{\tau}\right) & \text { if } m \text { is even, } \\ \operatorname{cl}\left(L * V_{\tau}^{-1}\right) & \text { if } m \text { is odd. }\end{cases}
$$

Moreover the $C_{m}^{a}$-equivalence is realized by surgery along simple $C_{m}^{a}$-trees with $r_{j} \geq$ $r_{j}\left(G_{\tau}\right)(j=1, \ldots, n)$.

(2) If $\varphi \in \mathcal{R}_{2 n-1}(k)$, then for an $n$-component string link $L$,

$$
\operatorname{cl}\left(L * V_{\varphi}\right) \stackrel{C_{2 n}^{a}}{\sim} \operatorname{cl}\left(L * V_{\varphi}^{-1}\right) .
$$

Moreover the $C_{2 n-1}^{a}$-equivalence is realized by surgery along simple $C_{2 n-1}^{a}$-trees with $r_{j} \geq r_{j}\left(G_{\varphi}\right)(j=1, \ldots, n)$. 


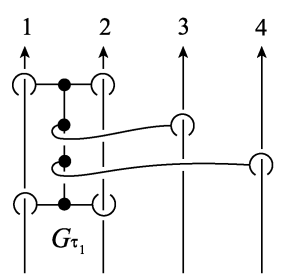

(a)

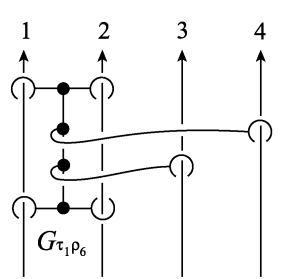

(b)



(c)

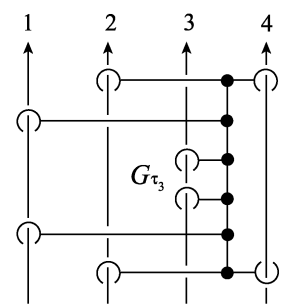

(d)

Figure 5.2. (a) $\tau_{1} \in \mathcal{P}_{6}(2)=\mathcal{P}_{2 n-2}(m-n)$, (b) $\tau_{1} \rho_{6} \in \mathcal{B}_{6}(2) \backslash$ $\mathcal{P}_{6}(2)$, (c) $\tau_{2} \in \mathcal{R}_{7}(3)=\mathcal{R}_{2 n-1}(n-1)$, and (d) $\tau_{3} \in \mathcal{R}_{8}(4)=$ $\mathcal{R}_{2 n}(n)$

Proof. (1) For $V_{\tau \rho_{m}}=\left(\mathbf{1}_{n}\right)_{G_{\tau \rho_{m}}}$, we can exchange the relative position of all pairs of leaves of $G_{\tau \rho_{m}}$ grasping the same component of $\mathbf{1}_{n}$ by, after taking closure, sliding the upper leaves along the orientation of $\operatorname{cl}(L)$. Lemmas 2.1, 2.2 and 2.4] imply that

$$
\operatorname{cl}\left(L * V_{\tau \rho_{m}}\right) \stackrel{C_{m}^{a}}{\sim} \operatorname{cl}\left(L *\left(\mathbf{1}_{n}\right)_{G}\right)
$$

where $G$ is obtained from $G_{\tau}$ by adding a positive half twist on each edge adjacent to a leaf (see Figure 5.3). Lemma 2.4 completes the proof.

The same arguments give us a proof of (2).

Now, for each $\varphi \in \mathcal{R}_{m}(k) \cup \mathcal{P}_{m}(k)$, we will calculate some Milnor invariants of the string link $V_{\varphi}$.
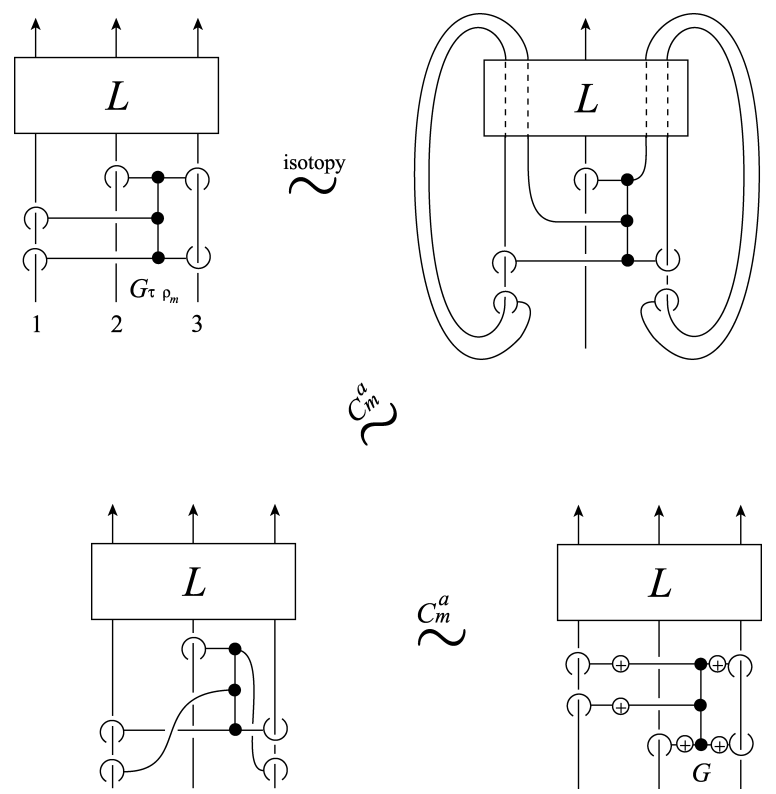

Figure 5.3 
Lemma 5.2. For $\varphi \in \mathcal{P}_{m}(k)(n+1 \leq m \leq 2 n, m-n \leq k \leq n)$ and for $\tau \in \bigcup_{l}\left(\mathcal{R}_{m}(l) \cup \mathcal{P}_{m}(l)\right)$,

$$
\mu_{\tau}\left(V_{\varphi}\right)= \begin{cases}1 & \text { if } \varphi=\tau \\ 0 & \text { if } \varphi \neq \tau\end{cases}
$$

We recall that $\mu_{\tau}\left(V_{\varphi}\right)=\mu_{V_{\varphi}}(\tau(1) \ldots \tau(m-2) l l)$ for $\tau \in \mathcal{R}_{m}(l) \cup \mathcal{P}_{m}(l)$.

Proof. We take the following 4 steps to prove this lemma.

Step 1. Make a new link $W_{\varphi}$ from $V_{\varphi}$ by taking parallels of the components of $V_{\varphi}$ so that Lemma 3.2 can be applied.

Consider the $C_{m-1}^{a}$-tree $G_{\varphi}$ for $\mathbf{1}_{n}$. Let $\tau \in \mathcal{R}_{m}(l) \cup \mathcal{P}_{m}(l)(m-n \leq l \leq n)$. Let $\mathbf{1}_{m}$ be the $m$-component trivial string link obtained from $\mathbf{1}_{n}$ by taking parallel copies of the components of $\mathbf{1}_{n}$ such that the $i$ th component of $\mathbf{1}_{m}$ is a parallel copy of either

$$
\left\{\begin{array}{l}
\text { the } \tau(i) \text { th component of } \mathbf{1}_{n} \text { if } i=1, \ldots, m-2, \text { or } \\
\text { the } l \text { th component of } \mathbf{1}_{n} \text { if } i=m-1, m,
\end{array}\right.
$$

and that $\mathbf{1}_{m}$ is contained in the tubular neighborhood $N\left(\mathbf{1}_{n}\right)$ of $\mathbf{1}_{n}$ with $G_{\varphi} \cap \mathbf{1}_{m} \subset$ $\operatorname{int}\left(G_{\varphi} \cap N\left(\mathbf{1}_{n}\right)\right)$. Since surgery along a $C_{m-1}$-tree preserves framings, the above correspondance can be naturally extended so that the $i$ th component of $\left(\mathbf{1}_{m}\right)_{G_{\varphi}}$ is a parallel copy of either

$$
\left\{\begin{array}{l}
\text { the } \tau(i) \text { th component of }\left(\mathbf{1}_{n}\right)_{G_{\varphi}} \text { if } i=1, \ldots, m-2 \text {, or } \\
\text { the } l \text { th component of }\left(\mathbf{1}_{n}\right)_{G_{\varphi}} \text { if } i=m-1, m .
\end{array}\right.
$$

Set $W_{\varphi}=\left(\mathbf{1}_{m}\right)_{G_{\varphi}}$. Since $G_{\varphi}$ is a $C_{m-1}^{a}$-tree, by Lemma 3.4. $\mu_{W_{\varphi}}(I)=\mu_{V_{\varphi}}(I)=0$ for any $I$ with $|I| \leq m-1$. Hence, by Lemma 3.2, we have

$$
\mu_{W_{\varphi}}(12 \ldots m)=\bar{\mu}_{\mathrm{cl}\left(W_{\varphi}\right)}(12 \ldots m)=\bar{\mu}_{\mathrm{cl}\left(V_{\varphi}\right)}(\tau(1) \ldots \tau(m-2) l l)=\mu_{\tau}\left(V_{\varphi}\right) .
$$

Step 2. By applying Lemma 2.6, deform $W_{\varphi}$ up to the $C_{m}$-equivalence into

$$
\left(\mathbf{1}_{m}\right)_{G_{\varphi_{1}}} *\left(\mathbf{1}_{m}\right)_{G_{\varphi_{2}}} * \cdots *\left(\mathbf{1}_{m}\right)_{G_{\varphi_{s}}}
$$

so that each $G_{\varphi_{j}}$ is a simple linear $C_{m-1}$-tree.

$$
\text { Set } V_{j}=\left(\mathbf{1}_{m}\right)_{G_{\varphi_{j}}}(j=1, \ldots, s) \text {. }
$$

Step 3. By Lemma 3.4, we have

$$
\mu_{W_{\varphi}}(12 \ldots m)=\mu_{V_{1} * \cdots * V_{s}}(12 \ldots m) .
$$

Since $\mu_{V_{j}}(I)=0$ for any $I$ with $|I| \leq m-1$, by Lemma 3.3 we have

$$
\mu_{W_{\varphi}}(12 \ldots m)=\mu_{V_{1}}(12 \ldots m)+\cdots+\mu_{V_{s}}(12 \ldots m) .
$$

Step 4. If $G_{\varphi_{j}}$ is a $C_{m-1}^{(2)}$-tree, then by Proposition 2.11, $V_{j}$ is link-homotopically trivial; hence $\mu_{V_{j}}(12 \ldots m)=0$. Otherwise, by using Lemma 4.1, calculate each $\mu_{V_{j}}(12 \ldots m)$. 
If $\left(\left|\varphi^{-1}(1)\right|, \ldots,\left|\varphi^{-1}(n)\right|\right) \neq\left(\left|\tau^{-1}(1)\right|, \ldots,\left|\tau^{-1}(n)\right|\right)$, then each $G_{\varphi_{j}}$ is a $C_{m-1}^{(2)}$-tree. This implies that $\mu_{W_{\varphi}}(12 \ldots m)=0$.

Suppose $\left(\left|\varphi^{-1}(1)\right|, \ldots,\left|\varphi^{-1}(n)\right|\right)=\left(\left|\tau^{-1}(1)\right|, \ldots,\left|\tau^{-1}(n)\right|\right)$. Since $\left|\varphi^{-1}(k)\right|=0$ and $\left|\tau^{-1}(i)\right| \geq 1(i \neq l)$, we have $k=l$, i.e., $\varphi \in \mathcal{P}_{m}(l)$.

If $\varphi \neq \tau$, then neither $(\varphi(1), \ldots, \varphi(m-1))$ nor $\left(\varphi \rho_{m}(1), \ldots, \varphi \rho_{m}(m-1)\right)$ is equal to $(\tau(1), \ldots, \tau(m-1))$. By Lemma 4.1, $\mu_{V_{j}}(12 \ldots m)=0$ for any $j(=1,2, \ldots, s)$.

If $\varphi=\tau$, then by Lemma 4.1 and by the fact that $\varphi \rho_{m} \neq \varphi$, there is a unique $C_{m-1}^{d}$-tree $G_{\varphi_{u}}$ in $\left\{G_{\varphi_{1}}, \ldots, G_{\varphi_{s}}\right\}$ such that

$$
\mu_{V_{j}}(12 \ldots m)= \begin{cases}1 & \text { if } j=u \\ 0 & \text { if } j \neq u\end{cases}
$$

This completes the proof.

Remark 5.3. The calculation method used in the proof of Lemma 5.2 can be applied in a more general context as follows. Let $T$ be a linear, simple $C_{m-1}$-tree for $\mathbf{1}_{n}$ whose ends both grasp the $k$ th component, and let $I=i_{1} \ldots i_{m-2} k k$ be a multi-index. Then, $\mu_{\left(\mathbf{1}_{n}\right)_{T}}(I)$ can be calculated as follows.

Step 1 . Make a new link $W=\left(\mathbf{1}_{m}\right)_{T}$ from $\left(\mathbf{1}_{n}\right)_{T}$ by taking parallels of the components of $\left(\mathbf{1}_{n}\right)_{T}$ so that Lemma 3.2 can be applied.

Step 2. By applying Lemma 2.6, deform $W$ up to the $C_{m}$-equivalence into $\left(\mathbf{1}_{m}\right)_{T_{1}}$ * $\left(\mathbf{1}_{m}\right)_{T_{2}} * \cdots *\left(\mathbf{1}_{m}\right)_{T_{s}}$ so that each $T_{j}$ is a simple linear $C_{m-1}$-tree.

Step 3. By applying Lemmas 3.4 and 3.3, we have

$$
\mu_{W}(12 \ldots m)=\mu_{\left(\mathbf{1}_{m}\right)_{T_{1}}}(12 \ldots m)+\cdots+\mu_{\left(\mathbf{1}_{m}\right)_{T_{s}}}(12 \ldots m) .
$$

Step 4. If $T_{j}$ is a $C_{m-1}^{(2)}$-tree, then by Proposition 2.11, $\mu_{\left(\mathbf{1}_{m}\right)_{T_{j}}}(12 \ldots m)=0$. Otherwise, by using Lemma 4.1, calculate each $\mu_{\left(\mathbf{1}_{m}\right)_{T_{j}}}(12 \ldots m)$.

Lemma 5.4. (1) For any $\varphi \in \mathcal{R}_{2 n-1}(k)(k=n, n-1)$, the Milnor invariants of $V_{\varphi}$ of length $\leq 2 n-1$ vanish.

(2) For $\varphi \in \mathcal{R}_{2 n-1}(n)$ and $\tau \in \mathcal{R}_{2 n}(n) \cup \mathcal{P}_{2 n}(n)$, there is a string link $U_{\varphi}$ such that $\operatorname{cl}\left(U_{\varphi}\right) \stackrel{C_{2 n-1}}{\sim} \operatorname{cl}\left(V_{\varphi}\right)$ and

$$
\left|\mu_{\tau}\left(U_{\varphi}\right)\right|= \begin{cases}1 & \text { if } \tau \in \mathcal{R}_{2 n}(n) \text { and } \varphi(i)=\tau(i)(i=1, \ldots, n-1), \\ 0 & \text { otherwise. }\end{cases}
$$

(3) For $\tau, \varphi \in \mathcal{R}_{2 n}(n) \cup \mathcal{P}_{2 n}(n)$,

$$
\mu_{\tau}\left(V_{\varphi}\right)= \begin{cases}1 & \text { if } \varphi=\tau \in \mathcal{P}_{2 n}(n), \\ 2 & \text { if } \varphi=\tau \in \mathcal{R}_{2 n}(n), \\ 0 & \text { if } \varphi \neq \tau .\end{cases}
$$

Remark 5.5. (1) Note that, for any $\varphi \in \mathcal{R}_{2 n-1}(n)$, there exists a unique element $\tau \in \mathcal{R}_{2 n}(n)$ such that $\varphi(i)=\tau(i)(i=1, \ldots, n-1)$, and that the correspondence induces a bijection from $\mathcal{R}_{2 n-1}(n)$ to $\mathcal{R}_{2 n}(n)$.

(2) For $\varphi \in \mathcal{R}_{2 n-1}(n-1)$ and $\tau \in \mathcal{R}_{2 n}(n) \cup \mathcal{P}_{2 n}(n)$, while the Milnor invariants of $V_{\varphi}$ of length $\leq 2 n-1$ vanish, $\mu_{\tau}\left(V_{\varphi}\right)$ is not easily calculated. However, we do not need the calculations to prove Theorem 1.3 . 


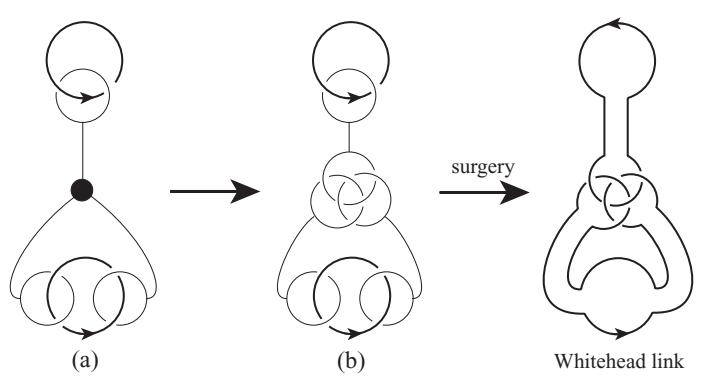

FiguRE 5.4. (a) 2-component trivial link with a simple $C_{2}$-clasper.

(b) 2-component trivial link with 3 basic claspers

Proof. As illustrated in Figure 5.4, the Whitehead link, which is a link $C(12,12)$ as defined in [2, subsection 7.11], is obtained from the 2-component trivial link by surgery along a simple $C_{2}$-tree. We recall that, for a sequence $i_{1} \ldots i_{k}$,

(i) $C\left(i_{1} i_{2}, i_{1} i_{2}\right)$ is a Whitehead link; and

(ii) $C\left(i_{1} \ldots i_{k} i_{k+1}, i_{1} \ldots i_{k} i_{k+1}\right)=K_{i_{1}} \cup \cdots \cup K_{i_{k}+1}$ is a $(k+1)$-component link obtained from $C\left(i_{1} \ldots i_{k}, i_{1} \ldots i_{k}\right)=K_{i_{1}} \cup \cdots \cup K_{i_{k-1}} \cup K_{i_{k}}^{\prime}$ by replacing $K_{i_{k}}^{\prime}$ by a Bing double $K_{i_{k}} \cup K_{i_{k+1}}$ of $K_{i_{k}}^{\prime}$.

A 4-component link obtained from the 4-component trivial link by surgery along the 11 basic claspers illustrated in Figure 5.5 is ambient isotopic to a link obtained by surgery along the 11 basic claspers of Figure 5.6, and to a link obtained from the trivial link by surgery along a clasper $H$ with boxes as illustrated in Figure 5.7 (for the definitions of a basic clasper and a box, see [11). Apply zip construction [11 twice to the clasper $H$ in Figure 5.7 the first one is for the marking $\left\{e_{1}\right\}$, and the second one is for $\left\{e_{2}\right\}$. Then $\operatorname{Zip}\left(\operatorname{Zip}\left(H,\left\{e_{1}\right\}\right),\left\{e_{2}\right\}\right)$ is $C_{7}$-equivalent to a link obtained by surgery along the $C_{6}$-tree of Figure 5.8. By Lemmas 2.4 and 5.1 (2), a link illustrated in Figure 5.8 is $C_{7}$-equivalent to $\operatorname{cl}\left(\left(\mathbf{1}_{4}\right)_{G_{\varphi}}\right)\left(=\operatorname{cl}\left(V_{\varphi}\right)\right)$, where $\varphi \in \mathcal{R}_{7}(4)=\mathcal{R}_{2 n-1}(n)$ is defined by $\varphi(1)=\varphi(5)=3, \varphi(2)=\varphi(4)=2, \varphi(3)=1$; see Figure 5.9. Since a link illustrated in Figure 5.6 is ambient isotopic to a link $C(1234,1234)$ (Figure 5.10); the link $C(1234,1234)$ is $C_{7}$-equivalent to $\operatorname{cl}\left(V_{\varphi}\right)$.

Similarly, we can see that, for $\varphi \in \mathcal{R}_{2 n-1}(k)(k=n, n-1)$, a link $C(\alpha, \alpha)$ $(\alpha=\varphi(n-1) \varphi(n-2) \cdots \varphi(1) k)$ is $C_{2 n-1}$-equivalent to $\mathrm{cl}\left(V_{\varphi}\right)$. In 2, subsection 7.11], it is shown that $\bar{\mu}_{C(\alpha, \alpha)}(I)=0$ for any $I$ with $|I| \leq 2 n-1$. Hence, by Lemma 3.4. we have the conclusion (1).

Let $U_{\varphi}$ be a band sum of $C(\alpha, \alpha)$ and $\mathbf{1}_{n}$ with $\operatorname{cl}\left(U_{\varphi}\right)=C(\alpha, \alpha)$; for example, see Figure 5.11, Let $\tau \in \mathcal{R}_{2 n}(n)$. Then it is not hard to see that $\operatorname{cl}\left(V_{\tau}\right)$ is ambient isotopic to a link $L((\bar{\beta}, \beta))(\beta=\tau(n-1)(\tau(n-2)(\cdots(\tau(1) n) \cdots)), \bar{\beta}=$ $((\cdots(n \tau(1)) \cdots) \tau(n-2)) \tau(n-1)$ defined in [2, subsection 7.4]. By combining [2, Proposition 6.5, Theorem 7.10 on p.42, and Theorem 7.10 on p.43], we have that if $\alpha=\beta$, i.e., $\varphi(i)=\tau(i)(i=1, \ldots, n-1)$, then

$$
\left|2 \mu_{U_{\varphi}}(I)\right|=\left|2 \bar{\mu}_{C(\alpha, \alpha)}(I)\right|=\left|\bar{\mu}_{L((\bar{\beta}, \beta))}(I)\right|
$$

for any $I$ with $|I|=2 n$. Then, (2) follows from (3). 
For $\tau, \varphi \in \mathcal{R}_{2 n}(n) \cup \mathcal{P}_{2 n}(n)$, by following the 4 steps in Remark [5.3], we have (3). This completes the proof.

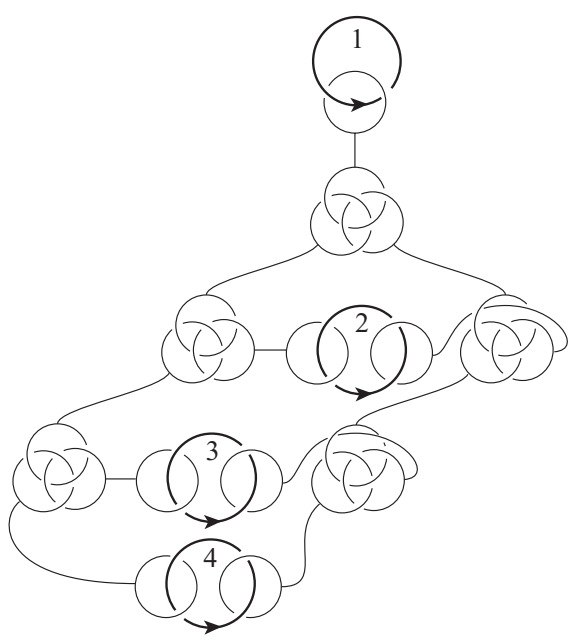

FiguRE 5.5. 4-component trivial link with 11 basic claspers. The numbers $1,2,3$, and 4 denote the order of the components

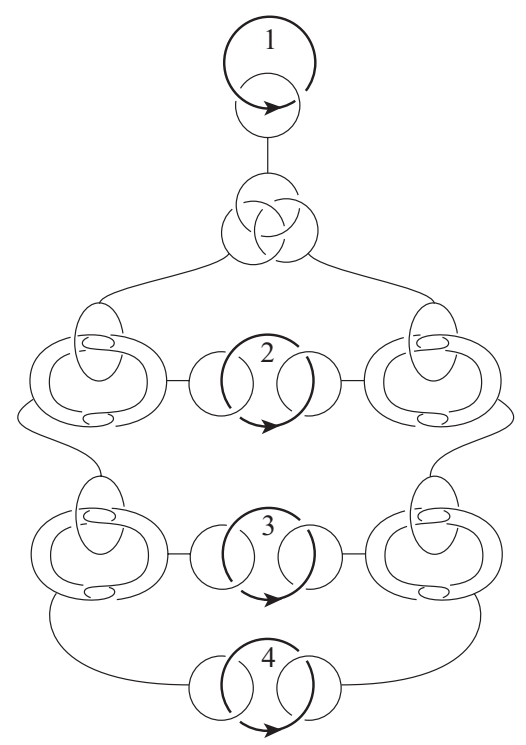

FiguRE 5.6 


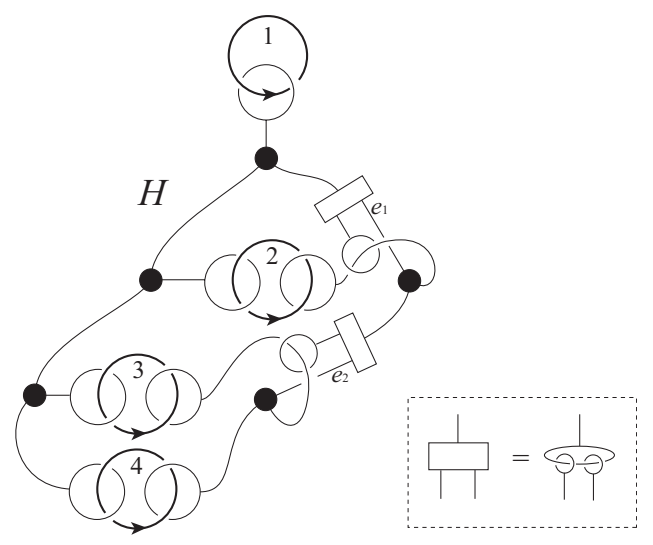

FIGURE 5.7

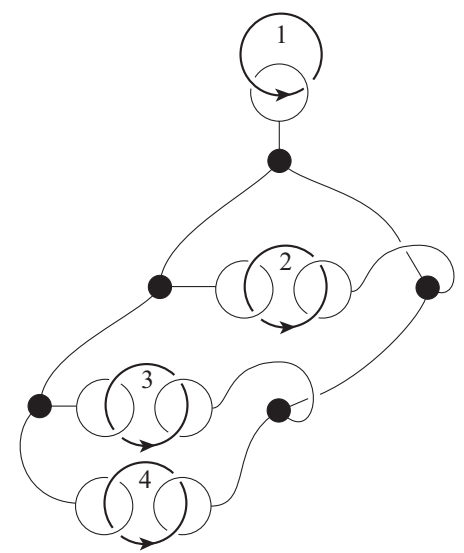

FiguRE 5.8

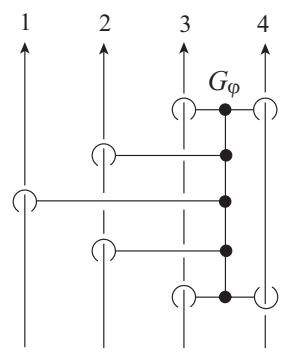

FIGURE 5.9. $\varphi \in \mathcal{R}_{7}(4)=\mathcal{R}_{2 n-1}(n) ; \varphi(1)=\varphi(5)=3, \varphi(2)=$ $\varphi(4)=2, \varphi(3)=1$

Lemma 5.6. Let $L$ be an $n$-component string link and $m(n+1 \leq m \leq 2 n)$ an integer. Let $T$ be a simple $C_{m-1}^{a}$-tree for $L$. Suppose that $\mu_{L}(I)=0$ for any $I$ with $|I| \leq m-1$ and $r(I) \leq 2$, and that $T$ is not a simple $C_{m-1}^{(3)}$-tree. Set 


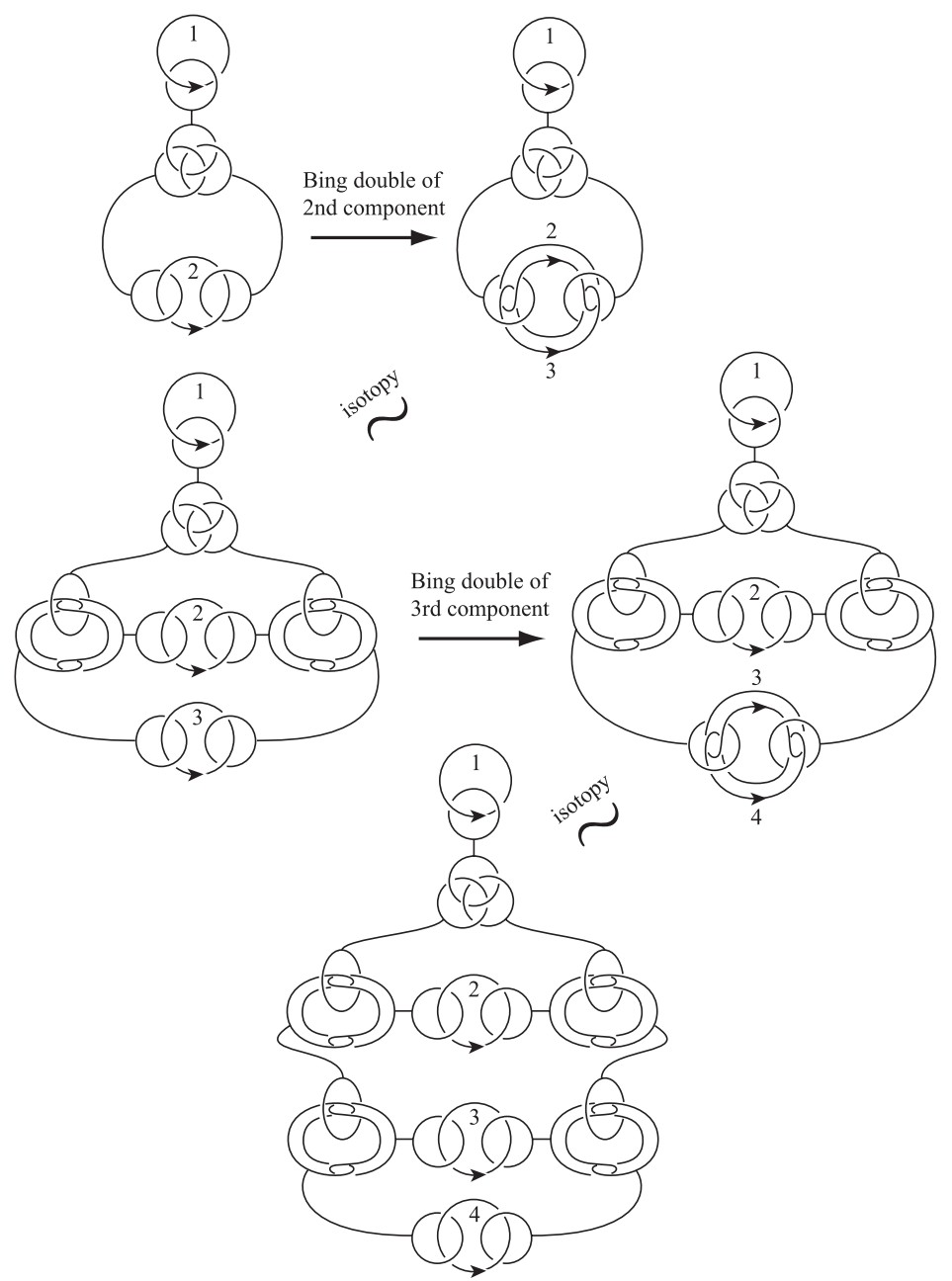

FIGURE 5.10. $\stackrel{\text { isotopy, }}{\sim}$ means that the links obtained by surgery along claspers are ambient isotopic

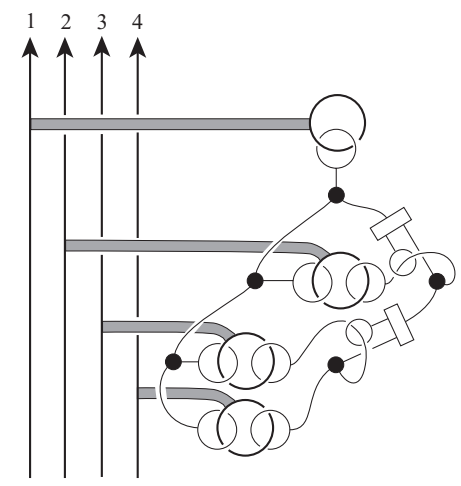

Figure 5.11. A band sum of $\mathbf{1}_{4}$ and $C(1234,1234)$ 
$k=\max \left\{i|| T \cap\left(i\right.\right.$ th component of $\left.\left.\mathbf{1}_{n}\right) \mid=2\right\}$. Then

(1) $\operatorname{cl}\left(L_{T}\right)$ is $C_{m}^{a}$-equivalent to $\operatorname{cl}\left(L * L^{\prime}\right)$, where $L^{\prime}=$

$$
\begin{cases}\prod_{\tau \in \mathcal{P}_{m}(k)} V_{\tau}^{\mu_{\tau}\left(L_{T}\right)-\mu_{\tau}(L)} & \text { if } n+1 \leq m \leq 2 n-2, \\ \prod_{\tau \in \mathcal{P}_{2 n-1}(k)} V_{\tau}^{\mu_{\tau}\left(L_{T}\right)-\mu_{\tau}(L)} * \prod_{\substack{\varphi \in \mathcal{R}_{2 n-1}(k) \\(\text { for some } \\ \varepsilon(\varphi) \text { 's in }\{0,1\})}} V_{\varphi}^{\varepsilon(\varphi)} & \text { if } m=2 n-1, \\ \prod_{\tau \in \mathcal{P}_{2 n}(n)} V_{\tau}^{\mu_{\tau}\left(L_{T}\right)-\mu_{\tau}(L)} * \prod_{\varphi \in \mathcal{R}_{2 n}(n)} V_{\varphi}^{\left(\mu_{\varphi}\left(L_{T}\right)-\mu_{\varphi}(L)\right) / 2} & \text { if } m=2 n,\end{cases}
$$

and

(2) the $C_{m}^{a}$-equivalence is realized by surgery along simple $C_{m}^{a}$-trees with $r_{k} \geq 2$.

Note that if $m \geq n+1$, then a simple $C_{m-1}^{a}$-tree is a simple $C_{m-1}^{(2)}$-tree. If $T$ is a simple $C_{m-1}^{(3)}$-tree, then by Proposition 2.11, $L_{T}$ and $L$ are self $\Delta$-equivalent.

Proof. By Lemma 2.1, $L_{T}$ is $C_{m}^{a}$-equivalent to $L *\left(\mathbf{1}_{n}\right)_{T^{\prime}}$, where $T^{\prime}$ is a simple $C_{m-1}^{a}$-tree and not a $C_{m-1}^{(3)}$-tree with

$$
\max \left\{i|| T^{\prime} \cap\left(i \text { th component of } \mathbf{1}_{n}\right) \mid=2\right\}=k \text {. }
$$

By induction on the length of the path connecting the two leaves grasping the $k$ th component, using Lemmas 2.5 and 2.4 we have that $\left(\mathbf{1}_{n}\right)_{T^{\prime}}$ is $C_{m}^{a}$-equivalent to a string link which is obtained from $\mathbf{1}_{n}$ by surgery along simple linear $C_{m-1}^{a}$-trees whose ends grasp the $k$ th component of $\mathbf{1}_{n}$. By Lemmas 2.1, 2.2, 2.4, we have that

$$
L_{T} \stackrel{C_{m}^{a}}{\sim} L * \prod_{\tau \in \mathcal{B}_{m}(k)} V_{\tau}^{u_{\tau}} .
$$

Moreover, by Lemmas 2.4 and 5.1, the closure $\operatorname{cl}\left(L * \prod_{\tau \in \mathcal{B}_{m}(k)} V_{\tau}^{u_{\tau}}\right)$ is $C_{m}^{a}$-equivalent to $\operatorname{cl}\left(L * L^{\prime}\right)$, where

$$
L^{\prime}= \begin{cases}\prod_{\tau \in \mathcal{P}_{m}(k)} V_{\tau}^{y_{\tau}} & \text { if } n+1 \leq m \leq 2 n-2, \\ \prod_{\tau \in \mathcal{P}_{m}(k)}^{y_{\tau}} V_{\tau}^{y_{\tau}} * \prod_{\varphi \in \mathcal{R}_{m}(k)} V_{\varphi}^{\varepsilon(\varphi)},(\varepsilon(\varphi) \in\{0,1\}) & \text { if } m=2 n-1, \\ \prod_{\tau \in \mathcal{P}_{m}(k)}^{y_{\tau}} V_{\tau}^{y_{\tau}} * \prod_{\varphi \in \mathcal{R}_{m}(k)}^{z_{\varphi}} V_{\varphi}^{z_{\varphi}} & \text { if } m=2 n .\end{cases}
$$

Note that the $C_{m}^{a}$-equivalences which are used in the above can be realized by surgery along simple $C_{m}^{a}$-trees with $r_{k} \geq 2$. By Lemmas 3.3, 5.2, and 5.4, for $\eta \in \mathcal{P}_{m}(k) \cup \mathcal{R}_{m}(k)$, we have that

$$
\mu_{\eta}\left(L * L^{\prime}\right)= \begin{cases}\mu_{\eta}(L)+y_{\eta} & \text { if } \eta \in \mathcal{P}_{m}(k), \\ \mu_{\eta}(L)+2 z_{\eta} & \text { if } m=2 n \text { and } \eta \in \mathcal{R}_{m}(k) .\end{cases}
$$

Since Milnor invariants of $L$ with length $\leq m-1$ and $r \leq 2$ vanish, by Lemma 3.4. those of $L_{T}$ also vanish. By combining this, the fact that $\operatorname{cl}\left(L_{T}\right)$ and $\operatorname{cl}\left(L * L^{\prime}\right)$ are $C_{m}^{a}$-equivalent, and Lemma 3.4, we have that

$$
\mu_{\eta}\left(L_{T}\right)=\bar{\mu}_{\eta}\left(\operatorname{cl}\left(L_{T}\right)\right)=\bar{\mu}_{\eta}\left(\operatorname{cl}\left(L * L^{\prime}\right)\right)=\mu_{\eta}\left(L * L^{\prime}\right) .
$$

This completes the proof. 
The following is the main result in this section.

Theorem 5.7. Let $L$ be an $n$-component Brunnian link. If $\bar{\mu}_{L}(I)=0$ for any $I$ with $|I| \leq 2 n-1$ and $r(I) \leq 2$, then $L$ is self $\Delta$-equivalent to the closure of $L^{\prime} * L^{\prime \prime}$, where

$$
L^{\prime}=\prod_{\varphi \in \mathcal{R}_{2 n-1}(n)} U_{\varphi}^{\varepsilon(\varphi)}, L^{\prime \prime}=\prod_{\tau \in \mathcal{R}_{2 n}(n)} V_{\tau}^{\left(\bar{\mu}_{\tau}(L)-\bar{\mu}_{\tau}\left(L^{\prime}\right)\right) / 2} * \prod_{\eta \in \mathcal{P}_{2 n}(n)} V_{\eta}^{\bar{\mu}_{\eta}(L)}
$$

and

$$
\varepsilon(\varphi)= \begin{cases}1 & \text { if } \bar{\mu}_{\tau}(L) \text { is odd for } \tau \in \mathcal{R}_{2 n}(n) \text { with } \tau(i)=\varphi(i)(i=1, \ldots, n-1), \\ 0 & \text { if } \bar{\mu}_{\tau}(L) \text { is even for } \tau \in \mathcal{R}_{2 n}(n) \text { with } \tau(i)=\varphi(i)(i=1, \ldots, n-1)\end{cases}
$$

Here $U_{\varphi}$ is the string link in Lemma 5.4 (2).

Note that, in the theorem above, $L^{\prime} * L^{\prime \prime}$ is determined by Milnor invariants of $L$ with length $2 n$ and $r=2$.

Proof. By Proposition 2.8, $L$ is obtained from the $n$-component trivial link $O$ by surgery along simple $C_{n-1}^{a}$-trees $T_{1}, \ldots, T_{l}$. Hence we have

$$
L=\operatorname{cl}\left(\left(\mathbf{1}_{n}\right)_{T_{1} \cup T_{2} \cup \cdots \cup T_{l}}\right) .
$$

By Lemmas 4.2, 2.1, 2.2 and 2.4, we have that

$$
L \stackrel{C_{n}^{a}}{\sim} \operatorname{cl}\left(\prod_{\pi \in \mathcal{F}_{n}} V_{\pi}^{\bar{\mu}_{\pi}(L)}\right)
$$

Since $\bar{\mu}_{\pi}(L)=0$ for any $\pi \in \mathcal{F}_{n}, L$ is $C_{n}^{a}$-equivalent to $O$; i.e., $L$ is obtained from $O$ by surgery along simple $C_{n}^{a}$-trees. By Lemmas 5.6 (1), 2.1, 2.2 and 2.4, we have that

$$
L \stackrel{C_{n+1}^{a}}{\sim} \operatorname{cl}\left(\prod_{1 \leq k \leq n}\left(\prod_{\tau \in \mathcal{P}_{n+1}(k)} V_{\tau}^{\bar{\mu}_{\tau}(L)}\right)\right)
$$

Since $\bar{\mu}_{\tau}(L)=0$ for any $\tau \in \mathcal{P}_{n+1}(k)(k=1, \ldots, n), L$ is $C_{n+1}^{a}$-equivalent to $O$. Note that a simple $C_{m}^{a}$-tree $(m \geq n+1)$ for an $n$-component link is a simple $C_{m}^{(2)}$-tree and might be a $C_{m}^{(3)}$-tree. By Proposition 2.11, surgery along a $C_{m}^{(3)}$-tree

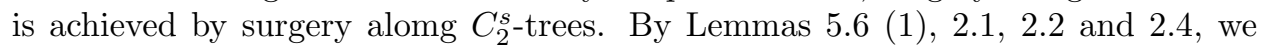
have that

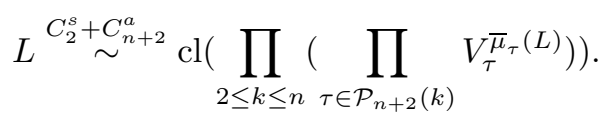

Since $\bar{\mu}_{\tau}(L)=0$ for any $\tau \in \mathcal{P}_{n+2}(k)(k=2, \ldots, n)$, by repeating this step, then by similar arguments and Lemma $5.1(2)$, we have that

$$
L \stackrel{C_{2}^{s}+C_{2 n-1}^{a}}{\sim} \operatorname{cl}\left(\prod_{\varphi \in \mathcal{R}_{2 n-1}(n)} V_{\varphi}^{\varepsilon(\varphi)} * \prod_{\phi \in \mathcal{R}_{2 n-1}(n-1)} V_{\phi}^{\varepsilon(\phi)}\right)
$$

for some $\varepsilon(\varphi)$ 's and $\varepsilon(\phi)$ 's in $\{0,1\}$.

In the proof of Lemma $5.4(1)$, we showed that, for $\phi \in \mathcal{R}_{2 n-1}(n-1)$,

$$
\operatorname{cl}\left(V_{\phi}\right) \stackrel{C_{2 n-1}}{\sim} C(\alpha, \alpha)
$$

where $\alpha=n \phi(n-2) \cdots \phi(1)(n-1)$. Since the Whitehead link $C(12,12)$ is deformed into a trivial link by a single self-crossing change in the first component, $C(\alpha, \alpha)$ is also deformed into a trivial link by a single self-crossing change in the $n$th component. So $C(\alpha, \alpha)$ is obtained from a trivial link by surgery along a simple $C_{1}^{s}$-tree $T$ 
with $r_{n}(T)=2$. By Lemma 2.9, $C(\alpha, \alpha)$ is obtained from a trivial link by surgery along simple $C_{n}^{a}$-trees with $r_{n}=2$. Since the Milnor invariants of $C(\alpha, \alpha)$ with length $\leq 2 n-1$ vanish, by similar arguments as above and Lemmas 5.6 (for $k=n$, $m \leq 2 n-1), 2.1$, 2.2, 2.4 and 5.1 (2), we have that

$$
C(\alpha, \alpha) \stackrel{C_{2}^{s}+C_{2 n-1}^{a}}{\sim} \operatorname{cl}\left(\prod_{\varphi \in \mathcal{R}_{2 n-1}(n)} V_{\varphi}^{\varepsilon^{\prime}(\varphi)}\right)
$$

for some $\varepsilon^{\prime}(\varphi)$ 's in $\{0,1\}$.

Since

$$
\prod_{\varphi \in \mathcal{R}_{2 n-1}(n)} V_{\varphi}^{\varepsilon(\varphi)} * \prod_{\phi \in \mathcal{R}_{2 n-1}(n-1)} V_{\phi}^{\varepsilon(\phi)}
$$

is obtained from $\mathbf{1}_{n}$ by surgery along simple $C_{2 n-2}^{a}$-trees, by Lemmas 2.1, 2.2, and 5.1 (2), $L$ is $\left(C_{2}^{s}+C_{2 n-1}\right)$-equivalent to the closure of a product of some $V_{\varphi}$ 's $\left(\varphi \in \mathcal{R}_{2 n-1}(n)\right)$. By Lemmas 2.4, 5.1 (2) and the proof of Lemma 5.4 (2), $L$ is $\left(C_{2}^{s}+C_{2 n-1}\right)$-equivalent to the closure of

$$
L^{\prime}=\prod_{\varphi \in \mathcal{R}_{2 n-1}(n)} U_{\varphi}^{\varepsilon^{\prime \prime}(\varphi)},
$$

where $U_{\varphi}$ is the string link in Lemma $5.4(2)$, and $\varepsilon^{\prime \prime}(\varphi)$ 's are integers in $\{0,1\}$.

Note that a simple $C_{2 n-1}$-tree for an $n$-component link is either a $C_{2 n-1}^{a}$-tree or a $C_{2 n-1}^{(3)}$-tree; hence by Proposition 2.11, $C_{2 n-1}$-equivalence implies $\left(C_{2}^{s}+C_{2 n-1}^{a}\right)$ equivalence. So $L$ is self $\Delta$-equivalent to a link obtained from $\operatorname{cl}\left(L^{\prime}\right)$ by surgery along simple $C_{2 n-1}^{a}$-trees. By Lemmas 5.6 (1) (for $m=2 n$ ), 2.1, 2.2 and 2.4, $L$ is $\left(C_{2}^{s}+C_{2 n}^{a}\right)$-equivalent to $\mathrm{cl}\left(L^{\prime} * L^{\prime \prime}\right)$, where

$$
L^{\prime \prime}=\prod_{\tau \in \mathcal{R}_{2 n}(n)} V_{\tau}^{\left(\mu_{\tau}(L)-\mu_{\tau}\left(L^{\prime}\right)\right) / 2} * \prod_{\eta \in \mathcal{P}_{2 n}(n)} V_{\eta}^{\mu_{\eta}(L)} .
$$

Since a $C_{2 n}$-tree is a $C_{2 n}^{(3)}$-tree, by Proposition 2.11 $L$ is self $\Delta$-equivalent to the closure of $L^{\prime} * L^{\prime \prime}$. By Lemmas 3.3, 3.4 and 5.4, we have that for any $\tau \in \mathcal{R}_{2 n-1}(n)$,

$$
\bar{\mu}_{\tau}(L) \equiv \varepsilon^{\prime \prime}(\varphi)(\bmod 2),
$$

where $\varphi(i)=\tau(i)(i=1, \ldots, n-1)$. This completes the proof.

By Theorem 5.7, we have the following two corollaries. Corollaries 5.8 and 5.9 are special cases of Theorem 1.3 and Corollary 1.5, respectively. Since these corollaries are needed to show Theorem 1.3, we give the statements.

Corollary 5.8. Let $L$ and $L^{\prime}$ be $n$-component Brunnian links. Suppose that $\bar{\mu}_{L}(I)$ $=\bar{\mu}_{L^{\prime}}(I)=0$ for any $I$ with $|I| \leq 2 n-1$ and $r(I) \leq 2$. Then $L$ and $L^{\prime}$ are self $\Delta$-equivalent if and only if $\bar{\mu}_{L}(J)=\bar{\mu}_{L^{\prime}}(J)$ for any $J$ with $|J|=2 n$ and $r(J)=2$.

Corollary 5.9. A Brunnian link $L$ is self $\Delta$-equivalent to a trivial link if and only if $\bar{\mu}_{L}(I)=0$ for any $I$ with $r(I) \leq 2$.

\section{LinKs WhOSE MiLnOR INVARIANTS VANiSH}

Before proving Theorem 1.3, we need some preparations.

Let $L=K_{1} \cup \cdots \cup K_{n}$ be an $n$-component link and $b$ a band attaching a single component $K_{i}$ in a way which is compatible with the orientations, i.e., $b \cap L=$ $K_{i} \cap b \subset \partial b$ consists of two arcs whose orientations from $K_{i}$ are opposite to those from $\partial b$. Then the $(n+1)$-component link $L^{\prime}=(L \cup \partial b) \backslash \operatorname{int}\left(b \cap K_{i}\right)$ is called a 
link obtained from $L$ by fission (along a band $b$ ), and conversely $L$ is called a link obtained from $L^{\prime}$ by fusion [14. For example, a ribbon link is a link obtained from a trivial link by a finite sequence of fusions.

Let $L^{\prime}=K_{11} \cup \cdots \cup K_{1 l_{1}} \cup \cdots \cup K_{n 1} \cup \cdots \cup K_{n l_{n}}$ be a link obtained from an $n$-component link $K_{1} \cup \cdots \cup K_{n}$ by a finite sequence of fissions, where $K_{i 1} \cup \cdots \cup K_{i l_{i}}$ is obtained from $K_{i}(i=1, \ldots, n)$. We assign a color $c\left(K_{i j}\right)$ to $K_{i j}$ as $c\left(K_{i j}\right)=i$. In this section, for a $C_{k}$-tree $T$, we call $T$ a $C_{k}^{s}$-tree (resp. $C_{k}^{d}$-tree) if $\mid\left\{c\left(K_{i j}\right) \mid T \cap\right.$ $\left.K_{i j} \neq \emptyset\right\} \mid=1$ (resp. $\left.=k+1\right)$. A $C_{k}^{s}$-move (resp. $C_{k}^{d}$-move) is a local move defined by surgery along a simple $C_{k}^{s}$-tree (resp. $C_{k}^{d}$-tree).

Lemma 6.1. If an n-component link is deformed into a trivial link by a finite sequence of fissions, $C_{2}^{s}$-moves and $C_{n-1}^{d}$-moves, then it is self $\Delta$-equivalent to a Brunnian link.

Proof. Note that $L$ is obtained from a trivial link by a finite sequence of fusions, $C_{2}^{s}$-moves and $C_{n-1}^{d}$-moves. By arguments similar to those in the proof of 11 , Proposition 3.22], we may assume that the bands of fusion, $C_{2}^{s}$-trees and $C_{n-1}^{d}$-trees are mutually disjoint. So there exist an $n$-component ribbon link $L_{0}$ and a disjoint union $F \cup F^{\prime}$ of simple $C_{2}^{s}$-trees and $C_{n-1}^{d}$-trees such that $L=L_{0 F \cup F^{\prime}}$, where $F$ (resp. $F^{\prime}$ ) is a disjoint union of $C_{2}^{s}$-trees (resp. $C_{n-1}^{d}$-trees). Since ribbon links are self $\Delta$-equivalent to a trivial link [25], $L_{0}$ is self $\Delta$-equivalent to the $n$-component trivial link $O$. Hence

$$
L \stackrel{C_{2}^{s}+C_{n-1}^{d}}{\sim} O
$$

This implies that $L$ is self $\Delta$-equivalent to a link obtained from $O$ by surgery along simple $C_{n-1}^{d}$-trees. Since a $C_{n-1}^{d}$-tree is a $C_{n-1}^{a}$-tree, by Proposition 2.8, we have the conclusion.

Theorem 6.2. Let $L$ be an n-component link such that $\bar{\mu}_{L}(I)=0$ for any $I$ with $|I| \leq 2 n-2$ and $r(I) \leq 2$. Then $L$ is self $\Delta$-equivalent to a Brunnian link.

Proof. Set $L=K_{1} \cup \cdots \cup K_{n}$. By Lemma6.1, it is enough to show that $L$ is deformed into a trivial link by a finite sequence of fissions, $C_{2}^{s}$-moves and $C_{n-1}^{d}$-moves.

Since any knot is $\Delta$-equivalent to an unknot [21], we may assume that every component of $L$ is trivial.

Suppose that any $k$-component sublink of $L$ is Brunnian $(2 \leq k \leq n-1)$. Since surgery along a simple $C_{k-1}^{d}$-tree with index $\left\{i_{1}, \ldots, i_{k}\right\}$ does not change the link type of a sublink $K_{j_{1}} \cup \cdots \cup K_{j_{k}}$ of $L$ for $\left\{j_{1}, \ldots, j_{k}\right\} \neq\left\{i_{1}, \ldots, i_{k}\right\}$, by Proposition 2.8, $L$ is $C_{k-1}^{d}$-equivalent to a link $L^{\prime}$ whose $k$-component sublinks are all trivial. Let $L_{0}$ be an $n$-component string link with $\operatorname{cl}\left(L_{0}\right)=L^{\prime}$, and set $\mathbf{1}_{n}=\gamma_{1} \cup \cdots \cup \gamma_{n}$. Let $\left\{S_{1}, \ldots, S_{m}\right\}\left(m=\left(\begin{array}{c}n \\ k\end{array}\right)\right)$ be the set of subsets of $\{1, \ldots, n\}$ with $\left|S_{i}\right|=k(i=1, \ldots, m)$. By Lemmas 2.1 and 2.2 ,

$$
L \stackrel{C_{k}}{\sim} \operatorname{cl}\left(L_{0} * L_{1} * \cdots * L_{m}\right)
$$

where, for each $i=1, \ldots, m, L_{i}=U_{i} \cup L_{i 1}$ is a split union of $U_{i}=\mathbf{1}_{n} \backslash \bigcup_{j \in S_{i}} \gamma_{j}$ and $L_{i 1}$ with $L_{i 1}=\left(\bigcup_{j \in S_{i}} \gamma_{j}\right)_{T_{i}}$ for some union $T_{i}$ of simple $C_{k-1}^{d}$-trees with index $S_{i}$, and the $C_{k}$-equivalence is realized by surgery along simple $C_{k}$-trees with $\mid$ index $\mid \geq k$. This implies that $L$ is $C_{k}^{d}$-equivalent to a link obtained from $\operatorname{cl}\left(L_{0} * L_{1} * \cdots * L_{m}\right)$ by surgery along simple $C_{k}$-trees with $\mid$ index $\mid=k$. 
By Lemmas 2.1 and 2.2 .

$$
L \stackrel{C_{k}^{d}+C_{k+1}}{\sim} \operatorname{cl}\left(L_{0} * L_{1}^{1} * \cdots * L_{m}^{1}\right)
$$

where, for each $i=1, \ldots, m, L_{i}^{1}=U_{i} \cup L_{i 1}^{1}$ is a split union of $U_{i}$ and $L_{i 1}^{1}$ with $L_{i 1}^{1}=\left(\bigcup_{j \in S_{i}} \gamma_{j}\right)_{T_{i}^{1}}$ for some union $T_{i}^{1}$ of simple $C_{k-1}^{d}$-trees and $C_{k^{-}}$-trees with index $S_{i}$, and the $C_{k+1}$-equivalence is realized by surgery along simple $C_{k+1}$-trees with $\mid$ index $\mid \geq k$. By Proposition [2.10, surgery along a simple $C_{k+1}$-tree with |index $\mid \geq k+1$ is realized by $C_{k}^{d}$-equivalence. Therefore, $L$ is $C_{k}^{d}$-equivalent to a link obtained from $\operatorname{cl}\left(L_{0} * L_{1}^{1} * \cdots * L_{m}^{1}\right)$ by surgery along simple $C_{k+1}$-trees with $\mid$ index $\mid=k$.

By Lemmas 2.1 and 2.2 .

$$
L \stackrel{C_{k}^{d}+C_{k+2}}{\sim} \operatorname{cl}\left(L_{0} * L_{1}^{2} * \cdots * L_{m}^{2}\right),
$$

where, for each $i=1, \ldots, m, L_{i}^{2}=U_{i} \cup L_{i 1}^{2}$ is a split union of $U_{i}$ and $L_{i 1}^{2}$ with $L_{i 1}^{2}=\left(\bigcup_{j \in S_{i}} \gamma_{j}\right)_{T_{i}^{2}}$ for some union $T_{i}^{2}$ of simple $C_{k-1^{-}}^{d}$-trees, $C_{k^{-}}$-trees, and $C_{k+1^{-}}$ trees with index $S_{i}$, and the $C_{k+2}$-equivalence is realized by surgery along simple $C_{k+2^{2}}$-trees with $\mid$ index $\mid \geq k$.

By repeating this procedure, we have that

$$
L \stackrel{C_{k}^{d}+C_{2 k}}{\sim} \operatorname{cl}\left(L_{0} * L_{1}^{k} * \cdots * L_{m}^{k}\right),
$$

where, for each $i=1, \ldots, m, L_{i}^{k}=U_{i} \cup L_{i 1}^{k}$ is a split union of $U_{i}$ and $L_{i 1}^{k}$ with $L_{i 1}^{k}=$ $\left(\bigcup_{j \in S_{i}} \gamma_{j}\right)_{T_{i}^{k}}$ for some union $T_{i}^{k}$ of simple $C_{k-1}^{d}$-trees, $C_{k}$-trees,..., and $C_{2 k-1}$-trees with index $S_{i}$. Note that a simple $C_{2 k}$-tree is either a $C_{2 k}$-tree with index $\geq k+1$ or a $C_{2 k}^{(3)}$-tree. By Propositions 2.10 and 2.11.

$$
L \stackrel{C_{k}^{d}+C_{2}^{s}}{\sim} \operatorname{cl}\left(L_{0} * L_{1}^{k} * \cdots * L_{m}^{k}\right) .
$$

So $L$ is self $\Delta$-equivalent to a link obtained from $\operatorname{cl}\left(L_{0} * L_{1}^{k} * \cdots * L_{m}^{k}\right)$ by surgery along simple $C_{k}^{d}$-trees. By Lemmas 2.1 and 2.2 .

$$
L \stackrel{C_{2}^{s}+C_{k+1}}{\sim} \operatorname{cl}\left(M_{1} * L_{0} * L_{1}^{k} * \cdots * L_{m}^{k}\right),
$$

where $M_{1}$ is a string link obtained from $\mathbf{1}_{n}$ by surgery along simple $C_{k}^{d}$-trees, and the $C_{k+1}$-equivalence is realized by surgery along simple $C_{k+1}$-trees with $\mid$ index $\mid \geq k+1$.

By Lemmas 2.1 and 2.2 ,

$$
L \stackrel{C_{2}^{s}+C_{k+2}}{\sim} \operatorname{cl}\left(M_{2} * L_{0} * L_{1}^{k} * \cdots * L_{m}^{k}\right),
$$

where $M_{2}$ is a string link obtained from $\mathbf{1}_{n}$ by surgery along simple $C_{k}^{d}$-trees and $C_{k+1}$-trees with $\mid$ index $\mid \geq k+1$, and the $C_{k+2}$-equivalence is realized by surgery along simple $C_{k+2}$-trees with $\mid$ index $\mid \geq k+1$.

By repeating this step, we have that

$$
L \stackrel{C_{2}^{s}+C_{2 n}}{\sim} \operatorname{cl}\left(M_{2 n-k} * L_{0} * L_{1}^{k} * \cdots * L_{m}^{k}\right),
$$

where $M_{2 n-k}$ is a string link obtained from $\mathbf{1}_{n}$ by surgery along simple $C_{k}^{d}$-trees, $C_{k+1}$-trees,..., and $C_{2 n-1}$-trees with $\mid$ index $\mid \geq k+1$. Since a simple $C_{2 n}$-tree is a $C_{2 n}^{(3)}$-tree, by Lemma 2.11

$$
L \stackrel{C_{2}^{s}}{\sim} \operatorname{cl}\left(M_{2 n-k} * L_{0} * L_{1}^{k} * \cdots * L_{m}^{k}\right) .
$$


Since $\left|S_{i}\right|=k$, the unions of the $j$ th components $\left(j \in S_{i}\right)$ of $\operatorname{cl}\left(M_{2 n-k} * L_{0} *\right.$ $\left.L_{1}^{k} * \cdots * L_{m}^{k}\right)$ and of $\operatorname{cl}\left(L_{0} * L_{i}^{k}\right)$ are equivalent. Let $L_{0 i}$ be the union of the $j$ th components $\left(j \in S_{i}\right)$ of $L_{0}$. Then $\operatorname{cl}\left(L_{0 i} * L_{i 1}^{k}\right)$ is deformed into a split sum of $\operatorname{cl}\left(L_{0 i}\right)$ and $\operatorname{cl}\left(L_{i 1}^{k}\right)$ by a finite sequence of fissions. Since the $k$-component sublinks of $\operatorname{cl}\left(L_{0}\right)\left(=L^{\prime}\right)$ are all trivial, $\operatorname{cl}\left(L_{0 i}\right)$ is the trivial link. Therefore $\operatorname{cl}\left(L_{0 i} * L_{i 1}^{k}\right)$ is concordant to $\operatorname{cl}\left(L_{i 1}^{k}\right)$. This implies that $\mu_{L_{i 1}^{k}}(J)=\bar{\mu}_{L}(J)=0$ for any multi-index $J$ in $S_{i}$ with $|J| \leq 2 k(\leq 2 n-2)$ and $r(J) \leq 2$. By Corollary [5.9] $\operatorname{cl}\left(L_{i 1}^{k}\right)$ is self $\Delta$-equivalent to a trivial link. Since $\operatorname{cl}\left(M_{2 n-k} * L_{0} * L_{1}^{k} * \cdots * L_{m}^{k}\right)$ is deformed into a split sum of $\operatorname{cl}\left(M_{2 n-k} * L_{0}\right)$ and $\operatorname{cl}\left(L_{11}^{k}\right), \ldots, \operatorname{cl}\left(L_{m 1}^{k}\right)$ by a finite sequence of fissions, $L$ is deformed into a split sum of $\operatorname{cl}\left(M_{2 n-k} * L_{0}\right)$ and a trivial link by a finite sequence of fissions and $C_{2}^{s}$-moves. Note that any $(k+1)$-component sublink of $\operatorname{cl}\left(M_{2 n-k} * L_{0}\right)$ is Brunnian.

By the induction, we have that $L$ is deformed into a split sum of an $n$-component Brunnian link $B$ and a trivial link by a finite sequence of fissions and self $\Delta$-moves. By Proposition 2.8, $B$ is $C_{n-1}^{d}$-equivalent to a trivial link. This completes the proof.

By combining Corollary 5.8 and Theorem 6.2, we can prove Theorem 1.3 ,

Proof of Theorem 1.3. Let $L$ be an $n$-component link with $\bar{\mu}_{L}(I)=0$ for any $I$ with $|I| \leq 2 n-1$ and $r(I) \leq 2$. By Theorem 6.2. $L$ is self $\Delta$-equivalent to a Brunnian link $B$. Since $\bar{\mu}_{B}(I)=\bar{\mu}_{L}(I)=0$ for any $I$ with $|I| \leq 2 n-1$ and $r(I) \leq 2$, by Corollary 5.8, $B$ is determined by Milnor invariants with length $2 n$ and $r=2$. This completes the proof.

The following theorem characterizes $n$-component links whose Milnor invariants of length $\leq 2 n-1$ and $r \leq 2$ vanish.

Theorem 6.3. For an $n$-component link $L, \bar{\mu}_{L}(I)=0$ for any $I$ with $|I| \leq 2 n-1$ and $r(I) \leq 2$ if and only if, for each $i \in\{1, \ldots, n\}$, there is a Brunnian link $L_{i}$ such that $L_{i}$ is self $\Delta$-equivalent to $L$ and the ith component $K$ of $L_{i}$ is null-homotopic in $S^{3} \backslash\left(L_{i}-K\right)$.

Proof. For the 'only if' part, it is enough to consider the case when $i=n$. By Theorem 6.2, $L$ is self $\Delta$-equivalent to a Brunnian link. In the proof of Theorem5.7, we see that a Brunnian link, whose Milnor invariants vanish for any $I$ with $|I| \leq$ $2 n-1$ and $r(I)$, is $\left(C_{2}^{s}+C_{2 n-1}\right)$-equivalent to the closure of a product of some $V_{\tau}$ 's $\left(\tau \in \mathcal{R}_{2 n-1}(n)\right)$. Hence, by Proposition 2.11 Lemmas 5.4 (1) and 5.6. $L$ is self $\Delta$-equivalent to $L_{n}$ of a product of some $V_{\varphi}$ 's $\left(\varphi \in \mathcal{R}_{2 n-1}(n) \cup \mathcal{R}_{2 n}(n) \cup \mathcal{P}_{2 n}(n)\right)$. Note that, for $\varphi \in \mathcal{R}_{2 n-1}(n)$ (resp. $\left.\varphi \in \mathcal{R}_{2 n}(n) \cup \mathcal{P}_{2 n}(n)\right) V_{\varphi}$ is $C_{2 n-1}^{(2)}$-equivalent (resp. $C_{2 n}^{(2)}$-equivalent) to $\mathbf{1}_{n}$ and the $C_{2 n-1}^{(2)}$-equivalence (resp. $C_{2 n}^{(2)}$-equivalence) is realized by surgery along simple $C_{2 n-1}^{(2)}$-trees (resp. $C_{2 n}^{(2)}$-trees) with $r_{n}=2$. By Proposition 2.11, $L_{n}$ is self $C_{1}$-equivalent to the trivial link and the self $C_{1}$ equivalence is realized by surgery along simple $C_{1}^{s}$-trees with $r_{n}=2$. Hence the $n$th component $K$ of $L_{n}$ is null-homotopic in $S^{3} \backslash\left(L_{n}-K\right)$.

Now we will show the 'if' part. Let $I$ be a multi-index with $|I| \leq 2 n-1$ and $r(I) \leq 2$. Since $L$ is self $\Delta$-equivalent to a Brunnian link, if $I$ does not contain an integer in $\{1, \ldots, n\}$, then $\bar{\mu}_{L}(I)=0$. So we may suppose that $I$ contains any integer in $\{1, \ldots, n\}$. The condition $|I| \leq 2 n-1$ implies that there is an integer $i$ such that $i$ appears in $I$ once. Let $L_{i}$ be a Brunnian link such that $L_{i}$ is self $\Delta$-equivalent to 




Figure 6.1

$L$ and the $i$ th component $K$ of $L_{i}$ is null-homotopic in $S^{3} \backslash\left(L_{i}-K\right)$. This implies that $\bar{\mu}_{L_{i}}(J i)=0$ for any index $J$ in $\{1, \ldots, n\} \backslash\{i\}$. Since $\bar{\mu}$ has 'cyclic symmetry' ([19, Theorem 8]), $\bar{\mu}_{L_{i}}(I)=0$. This completes the proof.

Example 6.4. Let $V$ be the string link illustrated in Figure 6.1 and let $L$ be the closure of $V$. By Proposition 2.11, for each $i(i=2,3), V$ is self $C_{1}$-equivalent to $\mathbf{1}_{3}$ and the self $C_{1}$-equivalence is realized by surgery along $C_{1}^{s}$-trees with index $\{i\}$. Hence the $i$ th component $K_{i}$ of $L$ is null-homotopic in $S^{3} \backslash\left(L-K_{i}\right)(i=2,3)$. Suppose that the 1st component $K_{1}$ is null-homotopic in $S^{3} \backslash\left(L-K_{1}\right)$. Then, by Theorem 6.3, $\bar{\mu}_{L}(I)=0$ for any $I$ with $|I| \leq 5$ and $r(I) \leq 2$. By Lemma 5.2, $\mu_{V}(12233)=1$. Hence $\bar{\mu}_{L}(12233)=1$. This is a contradiction.

\section{AKNOWLEDGMENTS}

The author would like to thank Thomas Fleming and Jean-Baptiste Meilhan for many useful discussions. He is also very grateful to Professor Tim Cochran for helpful comments. Finally, he wishes to thank the referee for his/her thoughtful reading.

\section{REFERENCES}

[1] A. J. Casson, Link cobordism and Milnor's invariant, Bull. London Math. Soc. 7 (1975), 39-40. MR0362286 (50:14728)

[2] T. Cochran, Derivatives of links: Milnor's concordance invariants and Massey's products, Mem. Amer. Math. Soc. 84 (1990), no. 427, x+73 pp. MR.1042041(91c:57005)

[3] J. Conant and P. Teichner, Grope cobordism of classical knots, Topology 43 (2004), 119-156. MR2030589 (2004k:57006)

[4] T. Fleming and A. Yasuhara, Milnor's invariants and self $C_{k}$-equivalence, Proc. Amer. Math. Soc. 137 (2009), 761-770. MR2448599

[5] T. Fleming and A. Yasuhara, Milnor numbers and the self delta classification of 2-string links, Intelligence of Low Dimensional Topology 2006, Knots and Everything Series, vol. 40, pp. 27-34, World Scientific, 2007. MR2371705

[6] C. H. Giffen, Link concordance implies link homotopy, Math. Scand. 45 (1979), 243-254. MR580602 (82a:57005)

[7] D. L. Goldsmith, Concordance implies homotopy for classical links in $M^{3}$, Comment. Math. Helv. 54 (1979), 347-355. MR.543335 (80h:57006)

[8] M.N. Gusarov, Variations of knotted graphs. The geometric technique of $n$-equivalence. (Russian), Algebra i Analiz 12 (2000), no. 4, 79-125; translation in St. Petersburg Math. J. 12 (2001), no. 4, 569-604. MR1793618 (2002g:57027)

[9] N. Habegger and X. S. Lin, The classification of links up to link-homotopy, J. Amer. Math. Soc. 3 (1990), 389-419. MR1026062 (91e:57015)

[10] K. Habiro, Master's thesis, University of Tokyo, 1994. 
[11] K. Habiro, Claspers and finite type invariants of links, Geom. Topol. 4 (2000), 1-83. MR1735632 (2001g:57020)

[12] K. Habiro, Brunnian links, claspers, and Goussarov-Vassiliev finite type invariants, Math. Proc. Cambridge Philos. Soc. 142 (2007), 459-468. MR2329695 (2008c:57022)

[13] K. Habiro and J.-B. Meilhan, Finite type invariants and Milnor invariants for Brunnian links, Int. J. Math. 19 (2008), 747-766. MR2431635(2009d:57022)

[14] A. Kawauchi, T. Shibuya and S. Suzuki, Descriptions on surfaces in four space, I. Normal forms, Math. Sem. Notes, Kobe Univ. 10 (1982), 75-125. MR672939 (84d:57017)

[15] J.-B. Meilhan, Invariants de type fini des cylindres d'homologie et des string links, Thèse de Doctorat (2003), Université de Nantes.

[16] J.-B. Meilhan, On surgery along Brunnian links in 3-manifolds, Alg. Geom. Topol. 6 (2006), 2417-2453. MR2286031 (2008h:57034)

[17] J.-B. Meilhan and A. Yasuhara, On $C_{n}$-moves for links, Pacific J. Math. 238 (2008), 119-143. MR 2443510

[18] J. Milnor, Link groups, Ann. of Math. (2) 59 (1954), 177-195. MR0071020 (17:70e)

[19] J. Milnor, Isotopy of links, Algebraic geometry and topology, A symposium in honor of S. Lefschetz, pp. 280-306, Princeton University Press, Princeton, N. J., 1957. MR 0092150 $(19: 1070 \mathrm{c})$

[20] H. A. Miyazawa and A. Yasuhara, Classification of n-component Brunnian links up to $C_{n}$ move, Topology Appl. 153 (2006), 1643-1650. MR2227018 (2007b:57013)

[21] H. Murakami and Y. Nakanishi, On a certain move generating link-homology, Math. Ann. 283 (1989), 75-89. MR995383 (90f:57007)

[22] Y. Nakanishi and Y. Ohyama, Delta link homotopy for two component links. III, J. Math. Soc. Japan 55 (2003), 641-654. MR1978214 (2004a:57009)

[23] Y. Nakanishi and T. Shibuya, Link homotopy and quasi self delta-equivalence for links, J. Knot Theory Ramifications 9 (2000), 683-691. MR.1762762 (2001d:57011)

[24] Y. Nakanishi, T. Shibuya and A. Yasuhara, Self delta-equivalence of cobordant links, Proc. Amer. Math. Soc. 134 (2006), 2465-2472. MR2213721 (2007a:57008)

[25] T. Shibuya, Self $\Delta$-equivalence of ribbon links, Osaka J. Math. 33 (1996), 751-760. MR 1424684 (97k:57012)

[26] T. Shibuya and A. Yasuhara, Self $C_{k}$-move, quasi self $C_{k}$-move and the Conway potential function for links, J. Knot Theory Ramifications 13 (2004), 877-893. MR2101233 (2006a:57009)

[27] T. Shibuya and A. Yasuhara, Boundary links are self delta-equivalent to trivial links, Math. Proc. Cambridge Philos. Soc. 143 (2007), 449-458. MR2364661

[28] N. Smythe, Boundary links, Topology Seminar (Univ. Wisconsin, Madison, Wis., 1965), pp. 69-72, Ann. of Math. Studies, No. 60, Princeton Univ.

[29] K. Taniyama and A. Yasuhara, Band description of knots and Vassiliev invariants, Math. Proc. Cambridge Philos. Soc., 133 (2002), 325-343. MR1912405 (2003m:57034)

Department of Mathematics, Tokyo Gakugei University, Koganeishi, Tokyo 184-8501, JAPAN

E-mail address: yasuhara@u-gakugei.ac.jp 